\title{
Defects in GaAs
}

\author{
V SWAMINATHAN \\ Bell Laboratories, Murray Hill, New Jersey 07974, USA
}

MS received 2 November 1981

\begin{abstract}
A comprehensive review of defects in GaAs with focus on native point defects and dislocations is given. The effects of these defects on devices are also considered. It is pointed out that a unified point defect model cannot at present be drawn from the available information. The importance of including anti-site defects in the point defect models which have hitherto only considered vacancies and interstitials is stressed. Attention is drawn to the need for understanding the dominant equilibrium native defects in $\mathrm{GaAs}$ both from fundamental and technological considerations. In this respect new experimental techniques are suggested to understand and control the defect structure. The current understanding of dislocations in GaAs is very much in its infancy compared to that in elemental semiconductors. Both theoretical work and careful experiments are wanting. This is essential since dislocations have been directly implicated in the degradation of GaAs devices.
\end{abstract}

Keywords. Native defects ; dislocations ; device degradation.

\section{Introduction}

Next to $\mathrm{Si}, \mathrm{GaAs}$ is the most important semiconducting material today. GaAs and related compounds such as $(\mathrm{Ga}, \mathrm{Al}) \mathrm{As}$ and $\mathrm{Ga}(\mathrm{As}, \mathrm{P})$ have received considerable attention in recent years because of their many potential applications. These applications include microwave devices, solar cells and several optoelectronic devices like light emitting diodes (LEDs), lasers, optoisolators, etc. With the refinements in crystal growth techniques, especially in epitaxial techniques which facilitate growing thin layers, the importance of this material has become more pronounced. As the demand for the material grew so did the demand for "perfect" material. More often than not poor devices result from poor starting material and the quality of the material is generally determined by the crystalline imperfections, which are invariably present. It has been the fond hope of both material scientists and device technologists to understand these imperfections and if possible to control them.

Crystalline imperfections include point defects, dislocations, stacking faults, grain boundaries, interfaces, etc. Foreign impurities are generally intentionally added to 
the material during crystal growth to obtain desired electrical properties. For example, in GaAs the group VI impurities - S, Se, Te-are used to acheive $n$-type conductivity while group II impurities - Be, $\mathrm{Mg}, \mathrm{Zn}$ - are used to get $p$-type conductivity. Even when the material is grown intentionally "undoped," satellite impurities such as $\mathrm{C}, \mathrm{Si}$ are present due to contamination from graphite crucibles and quartz containers used for crystal growth. As a result nominally undoped GaAs is never pure. Besides foreign impurities, point defects also include native defects which are vacancies (lattice sites empty of atoms), interstitials (atoms present at non-substitutional positions) and antisite defects (misplaced atoms). Thus for $\mathrm{GaAs}$ one can speak of $V_{\mathrm{Ga}}, V_{\mathrm{As}}, \mathrm{Ga}_{i}, \mathrm{Ga}_{\mathrm{As}}$ and $\mathrm{As} \mathrm{Ga}_{\mathrm{a}}$, where $V_{\mathrm{Ga}}$ is a vacancy at a gallium site, $\mathrm{Ga}_{i}$ is $\mathrm{Ga}$ atom occupying an interstitial site and $\mathrm{Ga}_{\mathrm{As}}$ is $\mathrm{Ga}$ atom occupying an arsenic site and so on.

Dislocations can be present in the as-grown material due to a variety of factors such as thermal stresses, excess point defects precipitating to form prismatic dislocation loops, etc. Dislocations are also introduced during the multifarious device processing steps like diffusion, ion implantation, sawing, polishing and so on. The presence of dislocations is, in general, harmful to the devices and often times the crystal grower aims to grow "low" dislocation density material. Apart from dislocations. two-dimensional defects such as stacking faults, grain boundaries and interfaces can also be present. For example, stacking faults are present in the asgrown material due to excess impurity atoms precipitating as "extrinsic" faults or introduced during processing steps such as diffusion and oxidation. Like dislocations, stacking faults also have deleterious effects on devices.

With the advancement in the growth of GaAs over the years, single crystal ingots of reasonable size are routinely grown such that grain boundaries are not problems any more. However, for some applications such as polycrystalline solar cells, grain boundary transistors, etc., grain boundaries play an important role.

For many device applications such as injection lasers and LEDs, GaAs is currently grown by epitaxial techniques. This has introduced another area of research namely understanding the interfaces of epitaxial layers. Another type of interface is that between the semiconductor and dielectric films $\left(\mathrm{Si}_{3} \mathrm{~N}_{4}, \mathrm{SiO}_{2}\right.$, etc.) which are deposited on GaAs during processing.

Current research on defects in GaAs is focussed on all of these imperfection impurities, native point defects, dislocations, stacking faults, grain and interface boundaries - with the aim to understand their nature and origin and to eliminate or control their density in the crystals. A review of all these defects is a Herculean task even if it were to be only on GaAs. For the sake of limiting the size of this review, focus is centred on native point defects and dislocations. In $\S 2$ we consider native point defects which is followed by $\S 3$ on dislocations. Section 4 discusses the effects of point defects and dislocations on devices. Section 5 summarizes the status of current understanding of this two class of defects in GaAs. Attention is drawn to the need for new tools to explore the GaAs defect structure.

\section{Native point defects}

\subsection{Definition}

Native point defects in GaAs can exist on both sublattices. Besides vacancies and interstitials, (sometimes referred in literature as self-interstitials to distinguish 
from foreign impurities occupying interstitial sites) in a compound $A B, A$ atoms can occupy sites belonging to $B$ atoms and vice versa giving rise to misplaced atoms (Kroger 1974 and Van Vechten 1975). Thus in GaAs, we have $V_{\text {As, }}$, $\mathrm{As}_{i}$ and $\mathrm{Ga}_{\mathrm{As}}$ in the As sublattice and $V_{\mathrm{Ga}}, \mathrm{Ga}_{i}$ and $\mathrm{As}_{\mathrm{Ga}}$ in the Ga-sublattice. These point defects can exist in neutral or charged form. The following notation has been proposed by Kroger and Vink (1956) to denote the charge state of the point defects ; $x$ denotes a neutral defect, prime denotes a defect with a negative charge and a dot denotes a defect with a positive charge. Thus $V_{\mathrm{Ga}}^{\prime \prime}$ is a doubly negatively charged gallium vacancy. The symbols used above actually represent effective charges rather actual charges of the defects. The difference in the charge states of a crystal site with and without an imperfection is defined as the effective charge. This distinction does not, however, apply for interstitial defects where the actual and effective charges are the same.

While the single substitutional defects $-V_{\mathrm{Ga}}, \mathrm{As}_{\mathrm{Ga}}, V_{\mathrm{As}}, \mathrm{Ga}_{\mathrm{As}}-$ retain $T_{d}$ symmetry in their unrelaxed positions, the symmetry of the interstitial defect depends on the particular configuration. The interstitial has the $T_{d}$ symmetry only if it occupies the centre of the unit cell (figure 1). In this configuration the interstitial is in an essentially nonbonding site. The interstitial can also exist in a bonded cofiguration. For example, a $\mathrm{Ga}_{\mathrm{i}}$ defect can nestle between a $\mathrm{Ga}$ and an As atom and form bonds with its two valence electrons in the bond-centred configuration. A third configuration is the split-interstitial configuration where two atoms occupy the same substitutional site. These three interstitial configurations are shown in figure 1. For a discussion of these interstitials in $\mathbf{S i}$, see the review by Corbett and Bourgoin (1975).

At present, no theoretical or experimental discussion of the different interstitial sites in GaAs exists. Of the three configurations one might expect the bond-centred configuration to be the likely one for small impurity interstitials such as $b_{i}{ }^{*}$ and body-centred one for the self-interstitials. But in both diamond and silicon, theoretical calculations suggest that the split $\langle 100\rangle$ interstitial is the lowest energy configuration for neutral as well as singly positively and singly negatively charged self-interstitial (Corbett and Bourgoin 1975). In view of some of the current point defect models in GaAs (Hurle 1979) which suggest interstitial defects to be one of the dominant native defects under equilibrium conditions both theoretical and experimental investigations of interstitials in GaAs would hopefully be initiated.

Besides single point defects, defect clusters would also occur. Such higher order defects would be particularly important in GaAs which is subjected to some radiation damage e.g. ion-implantation. Two examples for defect clusters are a divacancy $\left[\left(V_{\mathrm{Ga}}\right)_{2}\right.$ or $\left.\left(V_{\mathrm{As}}\right)_{2}\right]$ and prismatic dislocation loop which is formed as the result of aggregation of a large number of vacancies or interstitials.

\subsection{Charged point defects}

As we noted in the earlier section point defects can exist in either neutral or charged form. It is a priori difficult to predict what one should expect for the charge state 


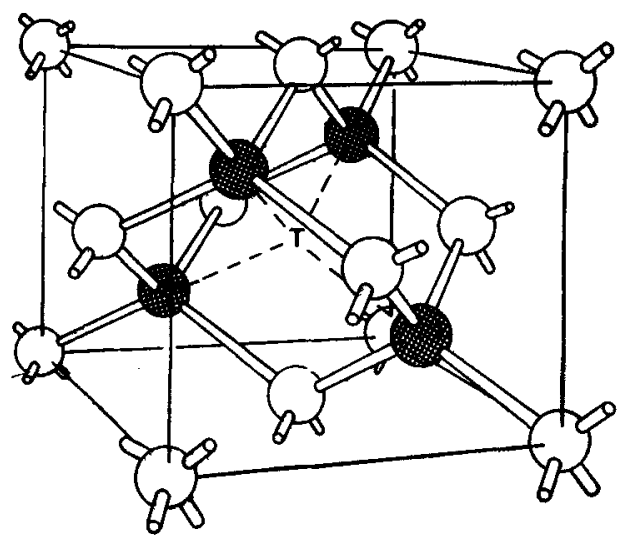

(a)

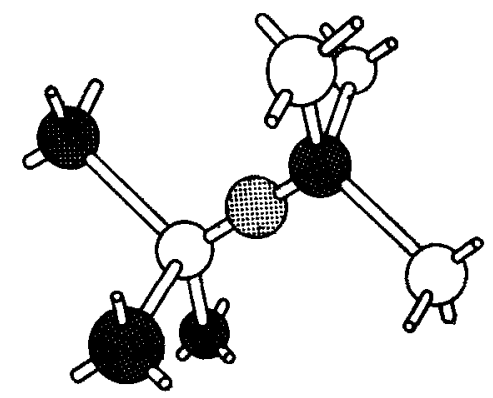

(b)

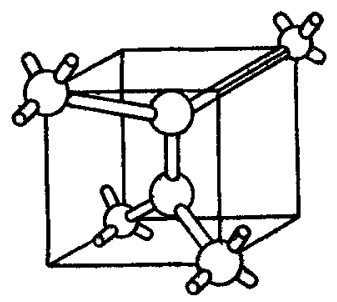

(c)

Figure 1. Interstitial configurations in GaAs: (a) tetrahedral configuration (b) bend - centered confizuration (c) $\langle 100\rangle$ split interstitial configuration (Corbett and Bourgoin 1975).

of a particular imperfection. Moreover, the charge state of the defect need not be the same under all conditions. For example, a vacancy in $\mathrm{Si}$ is known to exist in four forms- $V^{x}, V^{\prime}, V^{\prime}$ and $V^{\prime \prime}$-as $V^{\prime}$ in $p$-type $S i$ and as $V^{\prime}$ and $V^{\prime \prime}$ in $n$-type Si (Watkins 1975). Further recent refined theoretical calculations which take into account the lattice relaxation near the vacancy (Baraffet al 1980) showed that in $p$-type $\mathrm{Si}$, a doubly positively ionized vacancy, $V^{\cdot}$, has a stable level in the forbidden gap and that $V^{x}, V^{\prime}$ and $V^{*}$ form an "Andersen negative - $U^{\prime \prime}$ system. That is, the energy level of $V^{*}$ is in fact above that of $V^{\cdot}$ due to the large Jahn-Teller 
relaxation energy associated with $V^{x}$. In other words $V^{\cdot}$ is a metastable state and it would transform into $V^{x}$ and $V^{\cdot}$. Experimental results confirm this hypothesis (Watkins and Troxell 1980).

The understanding of point defects in GaAs is very much in its infancy compared to $\mathrm{Si}$. Both theoretical calculations and careful experiments are wanting. Notwithstanding these difficulties it is perhaps helpful to adopt certain general rules (Kroger 1974) regarding the charge state of the point defects in GaAs.

(i) Misplaced atoms - $\mathrm{As}_{\mathrm{Ga}}, \mathrm{Ga}_{\mathrm{As}}$ - would be expected to behave in the same way as a pentavalent impurity on a Ga site or a trivalent impurity on an As site. That is, $\mathrm{As}_{\mathrm{Ga}}$ would behave as a doubly ionizable donor and $\mathrm{Ga}_{\mathrm{As}}$ would behave as a doubly ionizable acceptor.

(ii) Interstitial atoms act as donors when their outer electron shell is less than half-full and as acceptors in the opposite case. Thus $\mathrm{Ga}_{i}$ would behave as a donor and $\mathrm{As}_{i}$ would behave as an acceptor in the tetrahedral sites.

(iii) Vacancies have donor action if the number of unpaired electrons near the vacancy is less than half the number of valence electrons present near the same site in the absence of vacancies. The opposite is true for the acceptor vacancy. Thus $V_{G_{a}}$ would behave as an acceptor and $V_{A s}$ would behave as a donor.

\subsection{Defect chemistry}

Native defects are introduced in GaAs when it is grown or when it is annealed after growth at high temperature and at some suitable partial pressure of one of the constituents, namely As in this case. After growth or annealing, when the crystals are somewhat rapidly cooled to room temperature, the high temperature equilibrium of native point defects is assumed to be frozen in. The concentration of point defects are then calculated by formulating quasi-chemical reactions involving the structure elements of the defects and atoms or molecules in outer phases and using the laws of mass action (Kroger 1974). The quasi-chemical approach is very helpful to calculate the variation in the concentration of point defects as a function of temperature and/or partial pressures of the dopants or one of the constituents of the compound and later compare the results with experimental data.

When defect calculations are performed some assumptions are made about the dominant native atomic disorder. There are three basic disorder mechanisms applicable to GaAs involving imperfections of the same nature:

(i) Schottky disorder involving $V_{\mathrm{Ga}}$ and $V_{\mathrm{As}}$.

(ii) Interstitial disorder involving $\mathrm{Ga}_{i}$ and $\mathrm{As}_{i}$.

(iii) Anti-structure disorder involving $\mathrm{Ga}_{\mathrm{As}}$ and $\mathrm{As}_{\mathrm{Ga}}$.

In order to maintain the $\mathrm{As} / \mathrm{Ga}$ site ratio required by the crystal structure, in a stoichiometric crystal the imperfections in (i), (ii), and (iii) occur in sets containing at least two types of them. Besides the three basic disorder mechanisms one can 


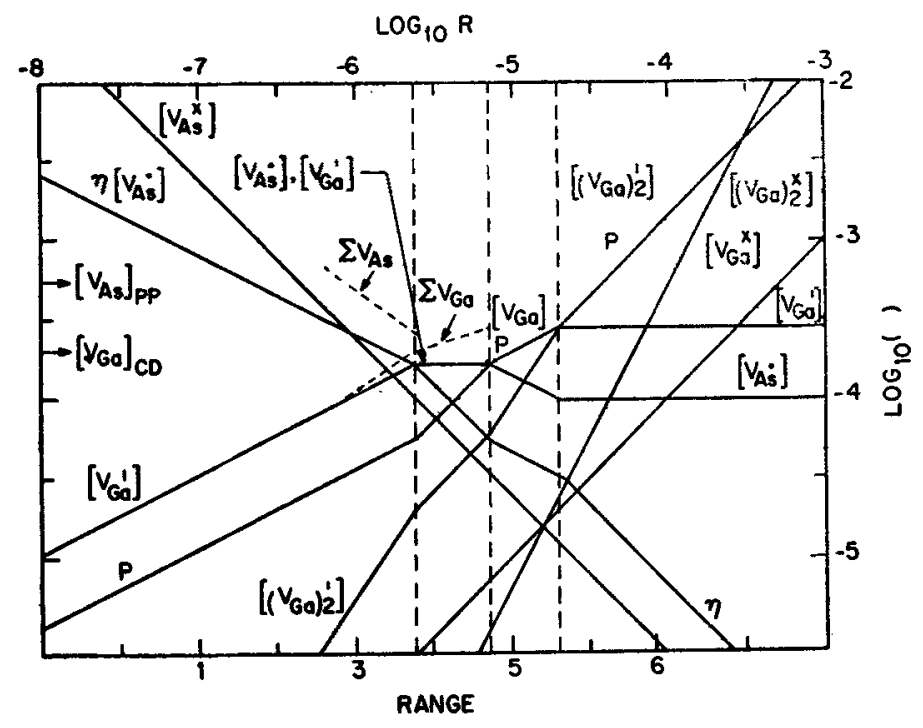

Figure 2. Defect concentrations at $1473 \mathrm{~K}$ as a function of parameter $R\left(\alpha P_{\mathrm{As}_{2}}^{1 / 2}\right)$. The quantities $\Sigma V_{A s}$ and $V_{G_{a}}$ are shown together with the experimental values [ $\left.V_{\mathrm{As}}\right]_{p p}$ and $\left[V_{\mathrm{Ga}}\right] C D$ (Logan and Hurle 1971).

have combinations of these. For example the Frenkel disorder involves $V_{\mathrm{Ga}}$ and $\mathrm{Ga}_{i}$ in the Ga-sublattice or $V_{\mathrm{As}}$ and $\mathrm{As}_{\mathrm{i}}$ in the As-sublattice. Thus there are nine possible types of disorder in GaAs.

Logan and Hurle (1971) first calculated the equilibrium concentration of point defects in $\mathrm{GaAs}$ as a function of temperature and arsenic pressure using the quasichemical approach. They assumed Schottky disorder mechanism as the dominant one based on the experimental data of Potts and Pearson (1966) and Chakraverty and Dreyfus (1966) available then. The concentration of the defects at $1200^{\circ} \mathrm{C}$ (0.98 $T_{M}$ where $T_{M}$ is the melting point in degrees Kelvin) calculated by Logan and Hurle as a function of a parameter which is proportional to $P_{A_{2}}^{1 / 2}$ is shown in figure 2. Note also that they have assumed that $V_{A s}$ behaves as a single donor and $V_{\mathrm{Ga}}$ as a single acceptor and that they have included $\left(V_{\mathrm{Ga}}\right)_{2}$ and $\left(V_{\mathrm{Ga}}\right)_{2}^{\prime}$ in the analysis.

Precision lattice parameter and density measurements on samples prepared under varying conditions have, however, cast doubt on the assumption that Schottky disorder was dominant. Principally, the lattice parameter measurements (Driscoll et al 1971; Driscoll and Willoughby 1972) indicated that the lattice parameter increases after annealing at high arsenic pressures. Similarly the density measurements of Bublik et al (1973) showed that on As rich side there is an excess mass per unit cell. Both these measurements suggested the existence of $\mathrm{As}_{i}$ or $\mathrm{As}_{\mathrm{Ga}}$ on the As rich side of GaAs. Based on this new data Hurle (1979) revised the earlier point defect model (Logan and Hurle 1971) by considering the Frenkel disorder in the As-sublattice $\left(\mathrm{As}_{1}, V_{\mathrm{As}}\right.$ ) as the dominant disorder. Hurle (1979) 
Table 1. Defect reactions and the corresponding mass action relations and estimated equilibrium constants for undoped GaAs (Hurle 1979)
(1) $0=e+h ; n p=k_{i}$
(2) $\mathrm{As}_{i} \mathrm{~s}+V_{i}=\mathrm{As}_{i}^{x}+V_{\mathrm{As}}^{x} ;[\mathrm{As} i]\left[V_{\mathrm{As}}\right]=K_{f a}$
(3) $\mathrm{As}_{i}^{x}=\mathrm{As}_{i}+e ;\left[\mathrm{A}_{s_{i}}\right] n /\left[\mathrm{As}_{i}^{x}\right]=K_{a i}$
(4) $V_{\mathrm{As}}^{x}=V_{\dot{A} s}+e ;\left[V_{\mathrm{As}}^{\cdot}\right] n /\left[V_{\mathrm{As}}^{x}\right]=K_{\mathrm{R},}$
(5) $\frac{1}{2} \mathrm{As}_{2}(\mathrm{~g})+V_{i}=\mathrm{As}_{i}^{x} ;\left[\mathrm{As}_{i}^{x}\right]=K_{\mathrm{As}_{i}} p_{\mathrm{As}_{2}}^{1 / 2}$

(1) $K_{l}=1.00 \times 10^{-12} T^{3} \exp (-1.52 / \mathrm{kT})^{a}$

(2) $K_{f a}=2.92 \times 10^{6} \exp (-4.85 / \mathrm{kT})$

(3) $K_{a i}=4.9 \times 10^{-9} T^{3 / 2} \exp (-? / \mathrm{kT})$

(4) $K_{\mathrm{av}}=442 \exp \left(-\frac{0.27}{\mathrm{kT}}\right)$

(5) $K_{A_{\mathrm{i}}}=16.4 \exp (-1 \cdot 13 / \mathrm{kT})$

Hurle used $1.62 \mathrm{eV}$ for $E_{q}$ which is incorrect.

also considered the incorporation of $\mathrm{Sn}, \mathrm{Te}$ and $\mathrm{Ge}$ in GaAs. Defect reactions and the estimated parameters of the corresponding mass-action constants considered by Hurle for undoped GaAs are given in table 1 .

\subsection{Experimental observation of point defects in GaAs}

Since the physical properties are greatly affected by imperfections, a variety of experiments have been performed with the aim to understand the point defect structure. These include self-diffusion experiments, Hall measurements, optical measurements (infrared absorption and photoluminescence), transient capacitance measurements, lattice parameter and density measurements and internal friction measurements. Table 2 lists the techniques that have been used in GaAs with some appropriate references.

Of the techniques that have been used in GaAs, electrical (Hall and conductivity) measurements and photoluminescence measurements are the two which are mostly commonly employed. Ikoma and Wang (1969) and Ikoma (1970) made Hall measurements on undoped GaAs in the temperature range 300-900 $\mathrm{K}$ and observed that the measured carrier concentration above $600^{\circ} \mathrm{K}$ was much in excess of the intrinsic carrier concentration. By fitting the temperature dependent Hall 
Table 2. Experiments performed to understand the defect structure of GaAs.

\begin{tabular}{ll}
\hline \multicolumn{1}{c}{ Experiment } & \multicolumn{1}{c}{ References } \\
\hline 1. Self-diffusion & Goldstein 1961, Palfrey and Willoughby 1980 \\
2. Hall, conductivity & Chiang and Pearson 1975a, Ikoma 1970 \\
3. Density & Bublik et al 1973 \\
4. Lattice parameter & Driscoll and Willoughby 1972 \\
5. Internal friction & Chakraverty and Drefyus 1966, Osvenskii, Kholodnyi \\
& and Milvidskii 1972 \\
6. Deep level transient spectroscopy & Lang, Logan and Kimerling 1977 \\
7. Photoluminescence & Williams and Bebb 1972 \\
8. Electron Paramagnetic Resonance & Wagner et al 1980 \\
9. Position lifetime & Gopinathan et al 1977 \\
10. Ballistic phonon attenuation & Narayanamurti, Logan and Chin 1979 \\
11. Mössbauer spectroscopy & Holm and Weyer 1980 \\
12. Transmission Electron Microscopy & Narayanan and Kachare 1975 \\
\hline
\end{tabular}

data these authors deduced in epitaxial $\mathrm{GaAs}$ an electron trap $\left(10^{19}-10^{20} \mathrm{~cm}^{-3}\right)$ with an activation energy of $0.25 \mathrm{eV}$ above the valence band which was suggested to be a native defect. Hurle (1979) hypothesized this defect to be $V_{\text {As }}$ acting as a donor. $\mathrm{He}$, however, noted that the high density of $V_{\text {As }}$ deduced by Ikoma and Wang was probably incorrect due to their omission in the analysis of the contribution from the substrates of the epitaxial wafers. Hurle (1979) further noted that in the high temperature Hall measurements of Roberts (1965) done in sealed ampoules with excess As, the observed intrinsic behaviour up to $1190^{\circ} \mathrm{C}$ was perhaps due to a low concentration of $V_{\mathrm{As}}$ and that $\mathrm{As}_{j}$ present under the experimental conditions were neutral. Based on the result that at high temperatures GaAs is extrinsic with the electron concentration probably equal to $\left[V_{\text {As }}\right]$, Hurle (1979) re-examined the solubility data for $\mathrm{Te}$ in GaAs in the light of his revised point defect model and deduced the ionization energy of $V_{\text {As }}$ to be $0.27 \mathrm{eV}$ (see table 1).

Chiang and Pearson (1975a) carried out high temperature annealing experiments under controlled arsenic pressures and observed that donors and acceptors are created by the treatments. The donor and acceptor were suggested to be $V_{\text {As }}$ and $V_{\mathrm{Ga}}$ since their concentrations varied as $p_{\mathrm{As}_{4}}^{-1 / 4}$ and $p_{\mathrm{As}_{4}}^{1 / 4}$ respectively. Photoluminescence measurements made concomitant with electrical measurements (Chiang and Pearson 1975b) suggested that both $V_{G a}$ and $V_{A s}$ are nonradiative. Chiang and Pearson (1975a) also deduced from their results the ionization energies of $V_{\text {As }}^{\circ}$ and $V_{\mathrm{Ga}}$ to be 0.7 and $0.4 \mathrm{eV}$ respectively. This is at variance with the value used by Hurle (1979). Further, according to Hurle's model one would expect that the donor concentration at room temperature due to $V_{\text {As }}$ should be high in undoped GaAs grown from Ga solution contrary to what is generally observed. For this 
reason Hurle (1979) proposed that the $V_{\mathrm{As}}$ disappear from the crystal on cooling by precipitating out from solution at some internal sink or by forming $\mathrm{Ga}_{\mathrm{As}}$. Chiang and Pearson (1975a) on the other hand observed donor behaviour after the crystals were cooled to room temperature. However, their results are not beyond doubt (Kroger 1977).

The assignment of donor action to $V_{\mathrm{As}}$ in the above papers is consistent with theoretical calculations on vacancies in GaAs. The essence of all the theoretical calculations is as follows. When a neutral atom, say As, is removed from the otherwise perfect crystal, $V_{\text {As }}$ that is formed is surrounded by the three electrons of the $\mathrm{Ga}$ atom. Similarly when $V_{\mathrm{Ga}}$ is formed, five of the As valence electrons are involved. The problem is then to find the electronic states that must be occupied by these "dangling" or unpaired electrons. The theoretical calculations that have been made so far, each following a slightly different approach arrive at the following result. There are two electronic states of $a_{1}$ and $t_{2}$ symmetry associated with a vacancy. For $V_{\mathrm{Ga}}$ which has 5 electrons, two are in $a_{1}$ state and the other three are in $t_{2}$ state. If spin degeneracy is included, the $t_{2}$ state can accept three more electrons and thus $V_{\mathrm{Ga}}$ acts as an acceptor. On the other hand the $t_{2}$ state of $V_{A s}$ is occupied by one electron and the remaining two electrons are in $a_{1}$ state. In this regard $V_{\mathrm{As}}$ acts as a donor. The energy levels of the donor and acceptor states, however, differ in these calculations. Il'in and Masterov (1976) obtained $0.25 \mathrm{eV}$ below the conduction band for the $V_{\mathrm{As}}$ donor level and $0.3 \mathrm{eV}$ above the valence band for the $V_{\mathrm{Ga}}$ acceptor level. Fazzio et al (1979) obtained $0.17 \mathrm{eV}$ and $0.55 \mathrm{eV}$ for the same defects. On the other hand Berholc and Pantelides (1978) and Jaros and Brand (1976) find the $t_{2}$ state of $V_{\mathrm{As}}$ to be slightly above and in resonance with the conduction band. It may be unrealistic to compare these theoretical calculations with experiments especially in view of the fact that the calculations have been made in the unrelaxed lattice without invoking effects of Jahn-Teller distortion. Such effects have been shown to have profound influence on the energy levels of vacancies in Si (Baraff et al 1980). Selfconsistent calculations of the energy levels of vacancies in GaAs similar to those used for silicon vacancy (Baraff et al 1980) have recently been initiated (Bachelet et al 1981).

In a few experiments acceptor action of $V_{\text {As }}$ has also been proposed. Munoz et al (1970) investigated the classic problem of type conversion observed in $n$-type GaAs when heat-treated at high temperatures $\left(760^{\circ} \mathrm{C}\right)$ as a function of arsenic pressure and found that acceptors are created at both high and low arsenic pressure as shown in figure 3 . They suggested that the acceptors are vacancies, $V_{\mathrm{As}}$ at low and $V_{\mathrm{Ga}}$ at high arsenic pressures respectively. Otsubo et al (1977) studied both bulk crystals and epitaxial films of $\mathrm{GaAs}$ annealed in an $\mathrm{H}_{2}$ flow by photoluminescence technique. They observed a new band at $1.39 \mathrm{eV}$ at $77^{\circ} \cdot \mathrm{K}$ after annealing. By noting that the amount of material near the surface which is damaged by annealing varies as the square root of heating time they concluded that diffusion limited process occurs at the heat-treated surface and $V_{A s}$ is the diffusing species. Further they suggested that the $1.39 \mathrm{eV}$ band is a donor-acceptor transition where the acceptor is suggested to be $V_{\text {As }}$.

Ikoma and Nakagawa (1972) considered the incorporation of amphoteric impurities in GaAs grown by vapour phase epitaxial techniques within the frame- 


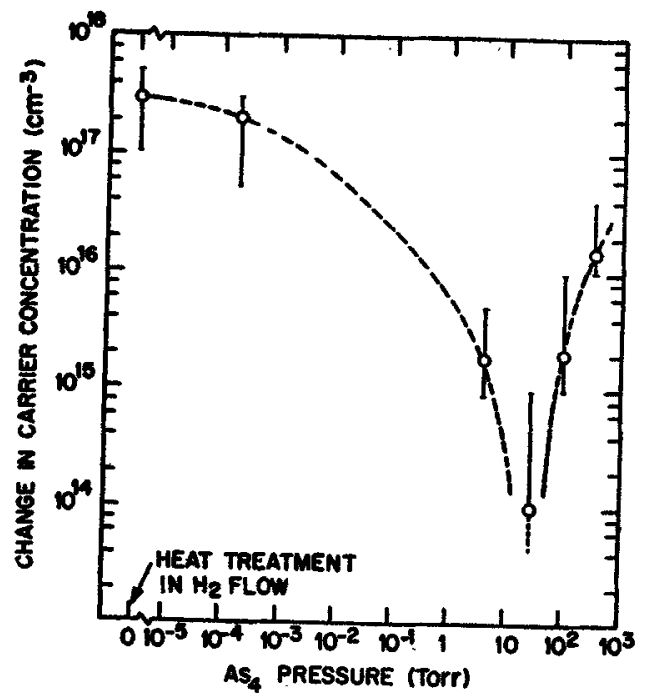

Figure 3. Change in surface carrier concentration in a variety of LPE GaAs crystals as a function of As4 pressure. Heat treatments were performed at $850^{\circ} \mathrm{C}$ for 30 min (Munoz et al 1970).

work of a model developed by Rytova and Fistul (1970) and found that the concentration of the compensating acceptors decreased with increasing partial pressure of arsenic. Therefore they suggested $V_{A s}$ to be the acceptors. Hasegawa and Majerfield (1976) associated a $0.64 \mathrm{eV}$ hole trap above the valence band to $V_{\text {As. }}$.

The assignment of acceptor behaviour to $V_{A s}$ is in apparent conflict with the result of Chiang and Pearson (1975a). Though $V_{A s}$ can be created under deficient As conditions the reasons given for the assignment of the acceptor to $V_{A s}$ in all these experiments are questionable. $\mathrm{Ga}_{\mathrm{As}}$ is also one of the possible point defects created under low As pressure. This defect is expected to behave as a double acceptor and could very well be responsible for the type conversion. Further the role of $\mathrm{Si}_{\mathrm{As}}$, which is a shallow acceptor, in these experiments cannot be excluded. $\mathrm{Si}$ is a common contaminant even in undoped $\mathrm{GaAs}$ because growth furnaces invariably contain quartz tubes. Under low As pressure $\mathrm{Si}_{\mathrm{As}}$ would be expected to form by the reaction of $\mathrm{Si}_{G a}$ with $V_{\mathrm{As}}$ (Spitzer and Allred 1968).

The role of native defects in GaAs has been emphasized in several other experiments where impurity-vacancy complexes have been invoked to explain the results. Photoluminescence has been one of the principal tools used in studying native defect complexes. The peak position of the bands seen predominantly in photoluminescence measurements and the suggested defect assignments are listed in table 3. It is clear that a comprehensive defect picture cannot at present be drawn from the photoluminescence measurements alone as evidenced by the conflicting assignments of the same emission band to more than one defect. The principal reason for this is that the crystals used by the various researchers are not of the same quality. Also the assignment of emission bands to single point defects is 
questionable as they are believed to be non-radiative. Chiang and Pearson (1975b) did not observe any new band assignable to vacancies after heat treatment. The decrease in photoluminescence intensity immediately following radiation damage is also believed to be due to the non-radiative nature of point defects (Arnold 1969; Jeong et al 1973; Swaminathan et al 1981b). Further, in annealing experiments the possibility of contamination by fast diffusing impurities such as $\mathrm{Cu}$ cannot entirely be ruled out.

Table 3. Peak positions of deep centre luminescence bands in $\mathrm{eV}$ and the suggested defect assignments in GaAs.

\begin{tabular}{|c|c|c|c|c|c|c|}
\hline \multicolumn{7}{|c|}{ Peak position (eV) in the range } \\
\hline \multirow{2}{*}{$\frac{0.5}{0.58 \mathrm{~V}_{\mathrm{Ga}}^{[1]}}$} & \multicolumn{2}{|r|}{$0.8-1 \cdot 0$} & \multirow{3}{*}{$\frac{1.2}{1.2 \mathrm{D}-\mathrm{V}_{\mathrm{Ga}}{ }^{(5)}}$} & \multicolumn{2}{|c|}{$1.34-1 \cdot 37$} & $1.39-1.41$ \\
\hline & 0.81 & $V_{\mathrm{Ga}}^{(1)}$ & & $1 \cdot 34$ & $\mathrm{Sn}-V_{\mathrm{As}}()$ & $V_{A s}^{(12)}$ \\
\hline & 0.93 & $\left(V_{\mathrm{Ga}} V_{\mathrm{As}}\right)^{2}$ & & $1 \cdot 35$ & $V_{\mathrm{Ga}}(1)$ & $1.39 \mathrm{D}-V_{\mathrm{As}}^{(13)}$ \\
\hline & 1.02 & $\left(V_{\mathrm{Ga}} V_{\mathrm{As}}\right)^{1}$ & & 1.35 & $\mathrm{Cu}-V_{\mathrm{As}}{ }^{(7)}$ & $1.41 \mathrm{Si}_{\mathrm{As}}-V_{\mathrm{As}}^{(14)}$ \\
\hline & 1.0 & $V_{\mathbf{G a}}{ }^{(3)}$ & & & $S i_{A s}-V_{A s}{ }^{(8)}$ & $1.41 \mathrm{C}_{A s}-V_{A s}{ }^{(15)}$ \\
\hline & $1 \cdot 0$ & $S i_{A s}-V_{A s}^{\left({ }^{(4)}\right.}$ & & & NiAs $1 \mathrm{As}$ & LAS CAS \\
\hline & $1 \cdot 0$ & $\mathrm{Cu}_{\mathrm{Ga}}-V_{\mathrm{As}}^{(2)}$ & & $1 \cdot 35$ & $V_{\mathrm{As}}(9,10)$ & \\
\hline & & & & $1 \cdot 37$ & $\mathrm{Cu}^{(1)}$ & \\
\hline & & & & $1 \cdot 37$ & $\mathrm{Zn}_{\mathrm{Ga}_{\mathrm{a}}}-V \mathrm{As}(\mathrm{t1})$ & \\
\hline & & & & & $\mathrm{Cd}_{\mathrm{Ga}}-V_{\mathrm{As}}$ & \\
\hline & & & & & $\mathrm{Cu}_{\mathrm{Ga}}-V_{\mathrm{As}}$ & \\
\hline & & & & & $\mathrm{Cu}_{\mathrm{Ga}_{\mathrm{a}}}-\mathrm{D}_{\mathrm{As}}$ & \\
\hline
\end{tabular}

1. Chang, Esaki and Tsu 1971

2. Vorobkalo et al 1973 ; Brailovskii, Groza and Broudnyi 1973

3. Pois and Pinard 1974

4. Batavin and Popova 1974

5. Williams and Bebb 1972

6. Nishizawa, Sinozaki and Ishida 1973

7. Glimchuk and Prokhorovich 1975

8. Birey and Sites 1980
9. Kryukova et al 1974

10. Lin et al 1975

11. Hwang 1968, 1969

12. Romano, Moran and Ashley 1973

13. Otsuba ef al 1977

14. Itoh and Takeuchi 1977

15. Lum et al 1977 

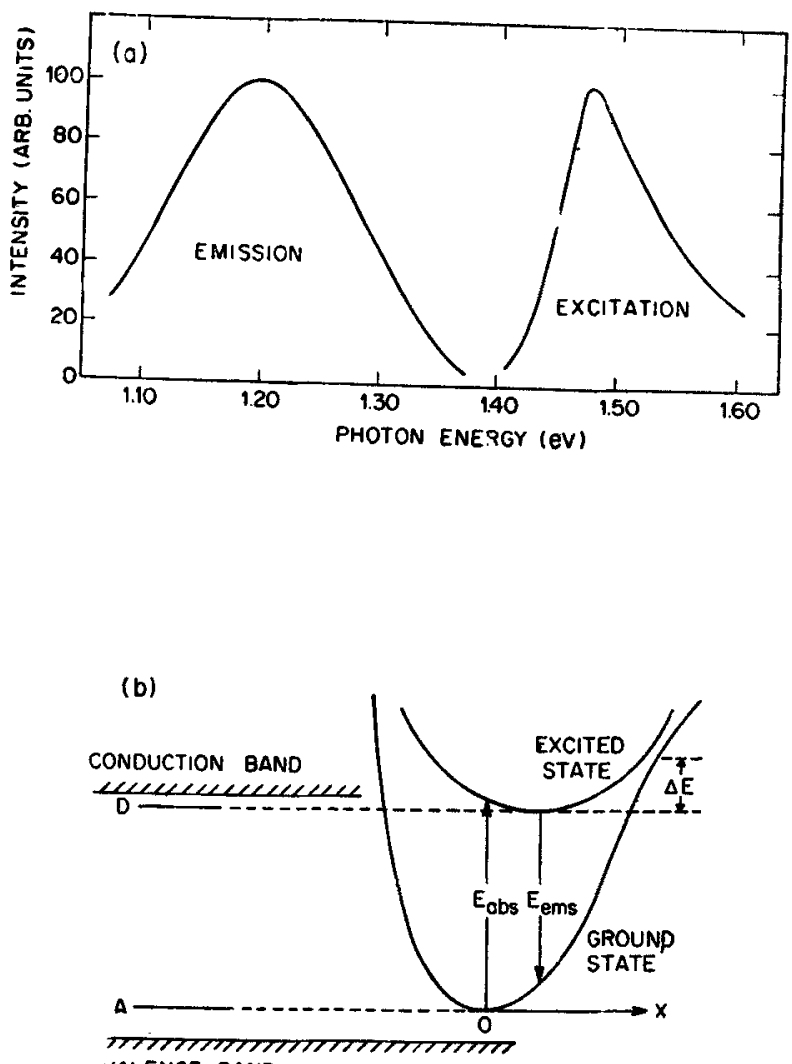

VALENCE BAND

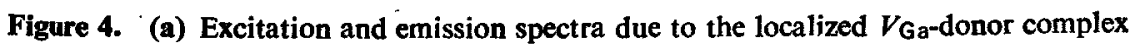
in Ge-doped GaAs. (b) The one-electron configurational coordinate model for vacancy complexes in GaAs. Also shown is the bandgap, and donor-like $(D)$ and acceptor-like $(A)$ levels of the zero-point energies of the ground state and excited states, respectively, which lie within the gap. $E_{\text {abs, }}$ the absorption energy, is greater than $E_{\text {ens }}$, the emission energy, and the difference is the Stokes shift. The electron transitions are vertical because of the Franck-Condon principle. $\Delta E$ is the activation energy for thermal quenching of the luminescence (Williams and Bebb 1972).

Of all the emission bands observed in GaAs, there is general consensus about the assignment of the $1.2 \mathrm{eV}$ band to donor $V_{\mathrm{Ga}}$ complex. This band is seen in all donor-doped samples and is weak in solution-grown GaAs. The latter supports the role of $V_{\mathrm{Ga}}$ since its concentration is expected to be low in solution grown material. Other features of this band are that it is broad with typical band widths of 150-200 MeV (Williams and Bebb 1972), that it is well separated from the band edge and is weak compared to the near band edge luminescence, and that it shows a large Stokes shift (figure 4a). The configurational coordinate model is invoked to explain this emission which is thought to be a localized transition in a donor- $V_{G a}$ molecule whose excited state is derived from the donor and the ground state from $V_{\mathrm{Ga}}$ (figure $4 \mathrm{~b}$ ). Bands having similar emission features have been 
observed in epitaxial $\mathrm{Ga}_{i-x} \mathrm{Al}_{x}$ As and impurity-vacancy complexes have been invoked. For example, a broad band observed at $1.55 \mathrm{eV}$ in Ge-doped $\mathrm{Ga}_{0.6} \mathrm{Al}_{0.4} \mathrm{As}$ grown by liquid phase epitaxy has been assigned to $\mathrm{Ge}_{\mathrm{As}}-V_{\mathrm{As}}$ complex (Swaminathan et al 1981b). Several broad bands observed in Sn-doped $\mathrm{Ga}_{1-x} \mathrm{Al}_{x}$ As grown by molecular beam epitaxy have been attributed to complexes involving $\mathrm{Sn}$ and $V_{\mathrm{Ga}}$ (Swaminathan and Tsang 1981c).

The emission band at $1.41 \mathrm{eV}$ has been studied quite extensively since it is observed after heat treatment in GaAs substrate used for growing epitaxial layers (Swaminathan et al 1981d). This band has been attributed to $\mathrm{Si}_{\mathrm{As}}-V_{\text {As }}$ (table 3). The participation of $\mathrm{Si}$ in this band is clear since it is observed only in Si-doped GaAs and further Swaminathan et al (1981) while identifying this band as due to donor-to-acceptor pair transition estimated the ionization energy of the acceptor to be $35 \mathrm{MeV}$ which is in agreement with the value for $\mathrm{Si}_{\text {As }}$ (Ashen et al 1975). The intensity of the band was found to decrease after As implantation (Itoh and Takeuchi 1977) and the band was absent after annealing under As-rich conditions (Rao and Duhamel 1978). Both these experiments suggested the role of a As deficient native defect $-V_{\mathrm{As}} \mathrm{Ga}_{\mathrm{As}}$ or $\mathrm{Ga}_{i}$ - in the band. A difficulty in choosing $V_{\text {As }}$ as the defect is the observation that the $1.41 \mathrm{eV}$ band is much narrower (typical band widths 25-50 MeV (Swaminathan et al 1981d)), than the $1.2 \mathrm{eV}$ band which has been assigned to donor- $V_{\mathrm{Ga}}$ complex. Since vacancy-related luminescence centres are expected to be broad and well separated from the band edge, as for example in the case of the $1.2 \mathrm{eV}$ band, perhaps $\mathrm{Ga}_{i}$ or impurity complex involving $\mathrm{Ga}_{i}$ might be the donor in the $1.41 \mathrm{eV}$ band (Swaminathan et al 1981d).

Impurity-vacancy complexes have also been invoked in other experiments. Hurle (1979) considered donor- $V_{\mathrm{Ga}}$ complexes as the compensating acceptors in Te and $\mathrm{Sn}$ doped $\mathrm{GaAs}$ and vapor grown Ge-doped GaAs (see also Kasano 1978). An electron trap at $0.83 \mathrm{eV}$ below the conduction band which has been observed in GaAs grown by vapour phase epitaxy (Miller et al 1977; Bhattacharya et al 1980) has been suggested to involve $V_{\mathrm{Ga}}$ because of the linear dependence of the trap on As/Ga ratio. Swaminathan and Copley (1975b) investigated the role of $\mathrm{Si}_{\mathrm{Ga}}-V_{\mathrm{Ga}}$ complexes in Si-doped $\mathrm{GaAs}$ and suggested the interaction between such complexes and mobile dislocations as being responsible for the hardening of GaAs containing donor impurities. The persistent photoconductivity observed in donor doped $\mathrm{Ga}_{1-x} \mathrm{Al}_{x}$ As has been suggested to arise from donor- $V_{\mathrm{As}}$ complexes (known as DX centres in literature) (Lang et al 1979; Lang and Logan 1979). A firm support for this assignment was obtained when different symmetries for the complex depending on whether the donor is on As-site or Ga-site were observed in measurements of attenuation of ballistic phonons (Narayanamurti et al 1979). $\mathrm{A} \mathrm{Te}_{\mathrm{As}}-V_{\mathrm{Ga}}$ complex having trigonal symmetry has been proposed in Te-doped GaAs from internal friction measurements (Osvenskii et al 1972).

The point defect structure in $\mathrm{GaAs}$ has also been investigated by studying the recovery of damage in samples which have been irradiated with some suitable high energy particles. We shall refer the reader to a recent review of this subject on III-V compounds (Lang 1976). Briefly, Lang et al (1977) investigated in detail by deep 


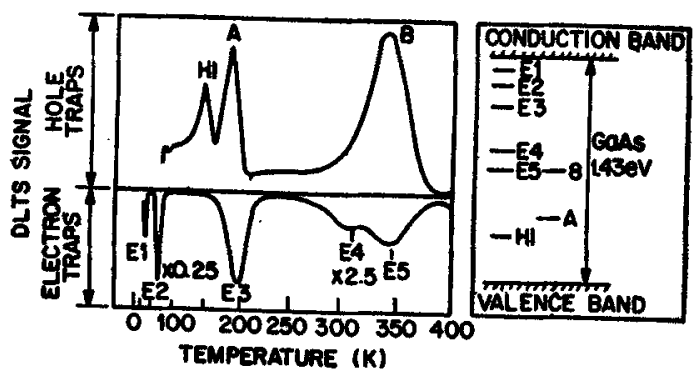

\begin{abstract}
Figure 5. Deep level transient spectroscopy spectrum of n-type GaAs irradiated at room temperature whth $1 \mathrm{MeV}$ electrons. Levels $A$ and $B$ ars present before irradiation. Irradiation induced levels are the five electron traps $E_{1}$ to $E_{5}$ and one hole traps $H_{1}$. The relative positions of the levels in the gap are shown in the inset on the right-hand side of the figure. Note the compressed scale for $E_{1}$ and $E_{2}$ and the expanded scale for $E_{4}$ and $E_{5}$ (Lang et al 1977).
\end{abstract}

level transient spectroscopy $\mathrm{GaAs}$ and $\mathrm{Ga}_{1-x} \mathrm{Al}_{x_{i}} \mathrm{As}$ irradiated with $1 \mathrm{MeV}$ electrons. They observed totally 6 energy levels, five electron traps and one hole trap after irradiation. The energy levels of these defects are shown in figure 5. The $E_{1}, E_{2}$ and $E_{3}$ levels were attributed to simple native defects. By studying the variation of these energy levels as a function of $x$ in $\mathrm{Ga}_{1-x} \mathrm{Al}_{x}$ As they suggested the $E_{3}$ level to be due to $V_{\mathrm{Ga}}$. This assignment was based on the observation that this level does not vary with $x$ meaning that it is tied to the valence band i.e. $V_{G}$ like behaviour. Recently, Pons et al (1980) have, however, disagreed with this assignment. They identified the $E_{2}$ level instead with the vacancy.

Indirect evidence for native defects and impurity vacancy complexes have also been provided by structural measurements in annealed $\mathrm{GaAs}$ by transmission electron microscope. Narayanan and Kachare (1975) investigated the microstructures of annealed heavily $\mathrm{Si}$ doped $\mathrm{GaAs}$. They observed prismatic dislocation loops in samples annealed in the range 600 to $850^{\circ} \mathrm{C}$ after a high temperature anneal at $1100^{\circ} \mathrm{C}$. They identified the prismatic loops to be of vacancy type. Based on the temperature dependence of the number and size of the loops they suggested $\left(\mathrm{Si}_{\mathrm{Ga}}-V_{\mathrm{Ga}}\right)$ complexes as the precursor defect to the vacancy loops. Prismatic loops observed in Li-diffused GaAs have also been identified to be vacancy type (Norris and Narayanan 1977).

Laister and Jenkins (1971) had earlier identified prismatic loops observed in annealed Te-doped GaAs to be vacancy type. Subsequently Hutchinson and 
Dobson (1974, 1975a) Morgulis et al (1974) and Wagner (1977) concluded that the prismatic loops in Te-doped $\mathrm{GaAs}$ and epitaxial $\mathrm{Ga}_{0.64} \mathrm{Al}_{0.36} \mathrm{As}$ (Wagner 1978) were actually of interstitial nature. While Morgulis et al assumed that the interstitial loops were formed by Te atoms. Hutchinson and Dobson suggested that they were formed by the native interstitials. Hatchinson and Dobson (1974) also found that after $1 \mathrm{MeV}$ electron irradiation and a $450^{\circ} \mathrm{C}$ anneal the prismatic dislocation loops grew which they considered as a support for the interstitial nature of the loop. This argument is not tenable for two reasons. irst, both interstitials' and vacancies would be created by $1 \mathrm{MeV}$ electron irradiation. Second, there has been no independent identification of interstitials even though vacancies have been attributed to the electron traps created by irradiation (Lang et al 1977; Pons et al 1980). Kamejima et al (1979) identified interstitial type prismatic loops in S-doped GaAs. Recently Hughes and Narayanan (1978) investigated Te-doped GaAs and disagreed with the interstitial identification of the prismatic loops and concluded that they were indeed vacancy type.

The resolution of this conflict between the vacancy and interstitial assignments of the prismatic dislocation loops observed in annealed $\mathrm{GaAs}$ is a crucial one. Hurle (1979) accepted the interstitial picture in developing the point defect model for Te-doped GaAs. The contrast analysis in the transmission electron microscope to determine whether a prismatic dislocation loop is interstitial type or vacancy type is unambiguous only when the loops are fairly large (loop diameter $>50 \AA$ ). In the above mentioned investigations the contrast analyses were performed on loops having diameter $100-500 \AA$ or greater and therefore experimenters' error is perhaps not the reason for the different results. A plausible reason could be differences in the starting material and/or the different methods of preparation of the samples for the annealing treatments. For example, while Hughes and Narayanan (1978) cleaned their samples and the quartz tubes used for the annealing experiments in aqueous $\mathrm{KCN}$ solution to reduce possible $\mathrm{Cu}$ contamination such a procedure was not followed by Hutchinson and Dobson $(1974,1975 a$.$) . It is worthwhile to mention here that the controversy of vacancy$ type versus interstitial type prismatic dislocation loops also exists in another III-V compound viz. GaP. Prismatic loops in S-doped GaP were identified as vacancy type by Stacy and DeKock (1978) and as interstitial type by Umeno et al (1979) and Ball and Hutchinson (1980).

In majority of experiments and theories invoking native defects, the anti-site disorder has not been considered at all. Van Vechten (1975) has calculated the formation energy of antisite disorder to be $6.1 \mathrm{eV}$ as compared to $5.2 \mathrm{eV}$ for the Schottky disorder or $5.6 \mathrm{eV}$ for the mixed antisite vacancy disorder. This suggests that the Schottky disorder might be more important than the antisite disorder. However, as we have noted earlier, the difficulty in assigning the acceptor created by heat-treating GaAs at low arsenic pressure to $V_{\text {As, }}$ in disagreement with the expected donor action for it, could easily be resolved by supposing that the acceptor is $\mathrm{Ga}_{\text {As. }}$. Moreover, recently Wagner et al (1980) have shown by high-field electron paramagnetic resonance the presence of $\mathrm{As}_{\mathrm{G}_{\mathrm{B}}}$. This new evidence necessitates the inclusion of antisite defects in the future point defect models. 
(0)

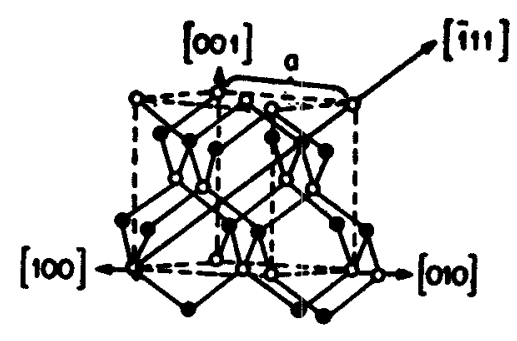

(b)

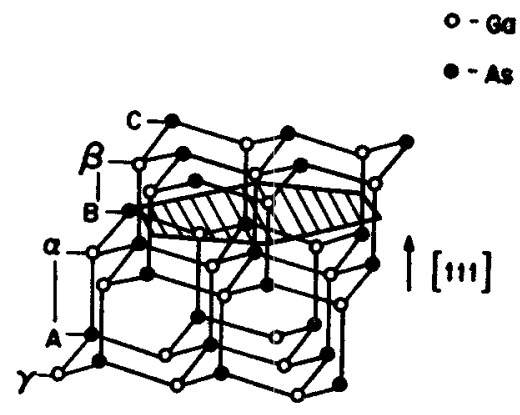

(c)

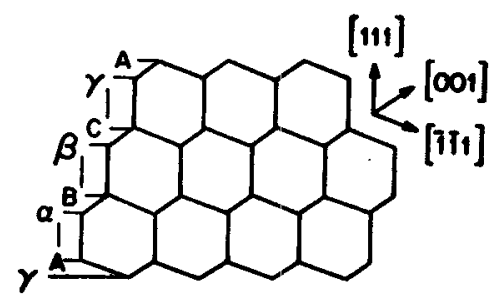

Figure 6. (a) The cubic unit cell of GaAs. (b) Stackinglof (111) planes. (c) Projection of figure $7 \mathrm{~b}$ on the (110) plane (Alexander and Haasen 1968).

\section{Dislocations}

\subsection{Perfect Dislocations}

GaAs crystallizes in the zinc blende structure which can be described as two interpenetrating fcc lattices. One lattice which is occupied by $\mathrm{Ga}$ atoms is shifted by $a / 4$ [111] relative to the other fcc lattice, which is occupied by As atoms, $a$ being the length of the fcc cube edge (figure 6a). The close packed $\{111\}$ planes of the fcc lattice become pairs of $\{111\}$ planes in GaAs as is shown in figure $6 \mathrm{~b}$ or in a two-dimensional projection on the $\{110\}$ plane in figure $6 \mathrm{c}$. Because of the presence of two type of atoms, there is a polarity in stacking of the $\{111\}$ planes. If $A B C A B C$ is the stacking order of $\{111\}$ planes in the first fcc sublattice and $\alpha \beta \gamma \alpha \beta \gamma$ that in the second, then $\alpha B, \beta C, \gamma A$ are closely spaced pairs of planes connected by three times as many bonds as are between the three times wider spaced $A \alpha, B \beta, C \gamma$ pairs of planes. 
(o)

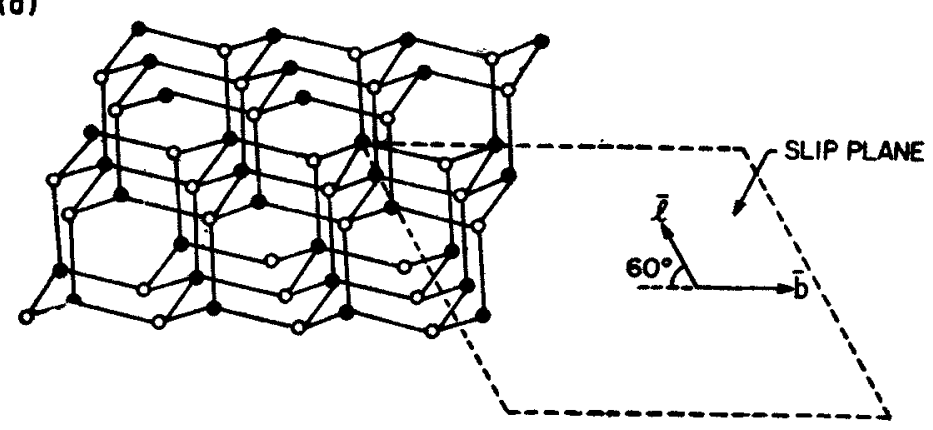

(b)

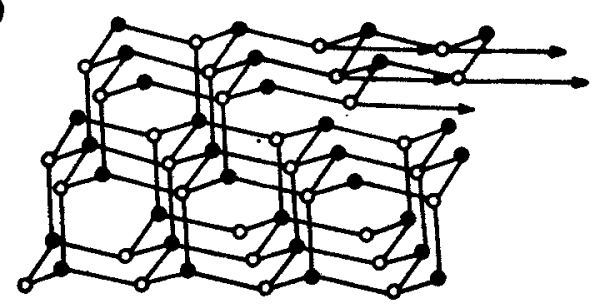

(c)

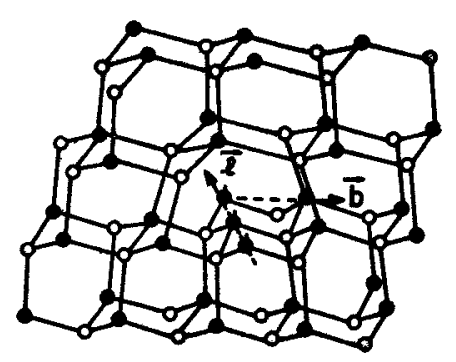

Figure 7. (a) The GaAs structure with a [111] direction vertical. A $\{111\}$ slip plane, the line direction and the Burgers vector of a $60^{\circ}$ dislocation are shown. (b) The result of moving a $60^{\circ}$ dislocation into the crystal from right to left is to produce the relative moments of the atoms indicated by the arrows. (c) The $60^{\circ}$ dislocation of the shuffle set in GaAs. $\bar{l}$ and $\bar{b}$ lie along $\langle 110\rangle$ directions. The slip plane is of (111\} type (Holt 1962).

Hornstra (1958) first examined the structure of a complete dislocation in crystals with diamond cubic structure and later Holt (1962) extended the analysis to the zinc blende structure. The important types of dislocations are the $60^{\circ}$ dislocation and the screw dislocation in the $\{111\}$ plane $\left(60^{\circ}\right)$ is the angle between the dislocation line and the Burgers vector (figure 7). Haasen (1957) pointed out that there are two types of $60^{\circ}$ ) dislocations in a compound semiconductor which he called $\alpha$ and $\beta$ dislocations. The $\alpha$ dislocation in $\mathrm{GaAs}$ ends with a row of $\mathrm{Ga}$ atoms, while the $\beta$ dislocation ends with a row of As atoms. The characteristics of complete dislocations having Burger's vector $a / 2[110]$ are shown in table 4. 
Table 4. Types of dislocations having Burgers vector $a / 2[110]$ in the zinc blende lattice (Hornstra 1958).

\begin{tabular}{ccccc}
\hline No. & $\begin{array}{c}\text { Symbol } \\
\text { of } \\
\text { axis }\end{array}$ & $\begin{array}{c}\text { Angle } \\
\text { between } \\
\text { axis and } \\
\text { Burgers } \\
\text { vector }\end{array}$ & $\begin{array}{c}\text { Glide } \\
\text { plane }\end{array}$ & $\begin{array}{c}\text { Number of } \\
\text { broken bonds } \\
\text { per cm }\end{array}$ \\
1. & $\langle 110\rangle$ & 00 & - & 0 \\
2. $\langle 110\rangle$ & $60^{\circ}$ & $\{111\}$ & 1.41 \\
3. $\langle 110\rangle$ & $90^{\circ}$ & $\{100\}$ & 2.83 or 0 \\
4. $\langle 211\rangle$ & $30^{\circ}$ & $\{111\}$ & 0.82 \\
5. $\langle 211\rangle$ & $90^{\circ}$ & $\{111\}$ & 1.63 \\
6. $\langle 211\rangle$ & $73^{\circ} 13^{\circ}$ & $\{311\}$ & 2.45 or 0.28 \\
7. & $\langle 211\rangle$ & $54^{\circ} 44^{\circ}$ & $\{110\}$ & 1.63 or 0 \\
8. & $\langle 100\rangle$ & $90^{\circ}$ & $\{110\}$ & 2.0 or 0 \\
9. & $\langle 100\rangle$ & $45^{\circ}$ & $\{110\}$ & 2.0 or 0 \\
\hline & & & & \\
\hline
\end{tabular}

One important aspect to recognize in the geometry of the $60^{\circ}$ dislocations in GaAs is that the extra half plane can terminate either between the $A \propto$ planes or between the $\alpha B$ planes. In the former case dislocation ends with a row of say, Ga atoms, while in the latter it ends with a row of As atoms. These two types of $60^{\circ}$ dislocations are referred in literature (Hirth and Lothe 1968) as the shuffle set (figure 7) and glide set (figure 8) respectively. A Ga dislocation in the shuffe set corresponds to an As dislocation in the glide set and vice versa. This is an important difference to bear in mind when the electrical properties of dislocations are discussed.

One easy way of characterizing dislocations in $\mathbf{G a} 1 \mathrm{~s}$ is to analyze the microstructure of deformed crysta!: wnere dislocations are cieliberately introduced. Table 5 shows the types of dislocations observed in the microstructure of Si-doped $n$-type $\left(n \sim 1.8 \times 10^{18} \mathrm{~cm}^{-3}\right) \mathrm{GaAs}$ that has been previously defon. at $550^{\circ} \mathrm{C}$ to a $3 \%$ strain (Swaminathan 1975). The microstructure analysis was performed in the transmission electron microscope where the Burgers vectors are 


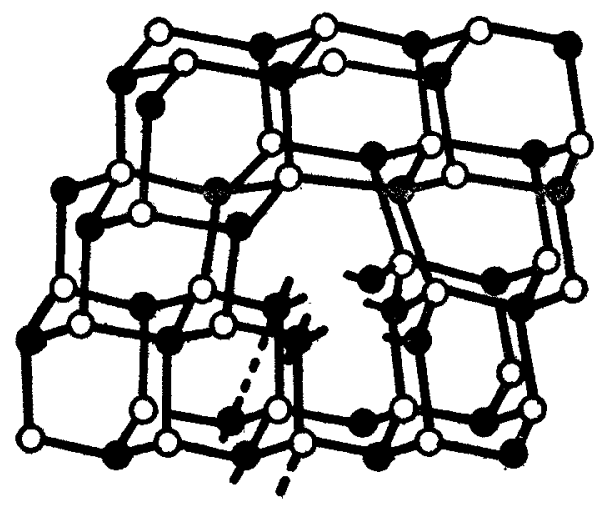

Figure 8. $60^{\circ}$ dislocation of the glide set in GaAs. The extra double plane is indicated by dashed lines. The dangling bonds are left dangling (Blanc 1975).

Table 5. Types of dislocations observed in GaAs deformed in compression at $550^{\circ} \mathrm{C}$ to strain varying from 3 to $20 \%$ (Swaminathan 1975 ).

\begin{tabular}{l|c|c|c}
\hline Slip plane & $\begin{array}{c}\text { Line direction } \\
\vec{l}\end{array}$ & $\begin{array}{c}\text { Angle between } \\
\bar{b}, \bar{l}\end{array}$ & $\begin{array}{c}\text { Number } \\
\text { seen }\end{array}$ \\
\hline $\overrightarrow{111})$ & {$[01 \overline{1}]$} & $60^{\circ}$ & 7 \\
$\overline{(111)}$ & $\overline{-121]}$ & $90^{\circ}$ & 6 \\
$(010)$ & {$[\overline{101]}$} & $90^{\circ}$ & 2 \\
$\overline{(131)}$ & {$[11 \overline{2}]$} & $73^{\circ}$ & 2 \\
\hline
\end{tabular}

determined by the standard methods of diffraction contrast analysis (Hirsch et al 1965). The Burgers vectors of all the dislocations determined by this method are of the type $a / 2\langle 110\rangle$. Most of the dislocations are the $60^{\circ}$ type though in some cases other types of dislocations are also observed. The dislocations are found to have $\{111\}$ plane which is the primary slip plane (Swaminathan 1975) in GaAs as well as $\{113\}$ and $\{100\}$. The dislocations on the $\{113\}$ planes may probably be grown-in dislocations. In some cases a Lomer dislocation with the slip plane $\{100\}$ is also seen. This is probably the result of the reaction of two glissile dislocations on different $\{111\}$ planes described by the equation

$$
a / 2[101]+a / 2[01 \overline{1}] \rightarrow a / 2[110]
$$


where the two dislocations on the left hand side of the equation glide in (11 $\overline{1})$ and (1 1 1) planes respectively. The produced Lomer dislocation lies in (001) plane.

A characteristic feature of the microstructure of deformed $\mathrm{GaAs}$ is the presence of long straight dislocations aligned along $\langle 110\rangle$ and $\langle 112\rangle$ directions as shown in figure 9. The alignment of dislocations along $\langle 110\rangle$ and $\langle 112\rangle$ has been found in other semiconduetors as well and it cannot be ascribed to elastic anisotropy. Table 6 shows that covalent materials are no less isotropic than fcc metals in which the phenomenon does not occur. Further it is not obvious that the $\langle 110\rangle$ and $\langle 112\rangle$ directions will be low core energy directions. The alignment is presumably due to the low Peierls energy along these directions. The alignment of dislocations along the $\langle 110\rangle$ and $\langle 112\rangle$ directions disappears, however, with increasing percentage of deformation as shown in figure 10 where the microstructure resembles that of fcc metals.

Though the primary slip plane in GaAs is the $\{111\}$ plane one can distinguish two types of $\{111\}$ planes in the zinc blende structure. These are the $A \alpha$ and $\alpha B$ planes in figure 6 . Since glide in the widely-spaced $A \alpha$ planes involves breaking of the smallest number of bonds, they were suggested to be the slip planes (Shockley

Table 6. Elastic isotropy of fcc metals and of diamond and of zinc blende structure semiconductors. The criterion for elastic isotropy is $\mathrm{C}_{11}-\mathrm{C}_{12}=2 \mathrm{C}_{44}$.

\begin{tabular}{cc}
\hline Material & $\frac{C_{11}-C_{12}}{2 C_{44}}$ \\
\hline Al & 0.82 \\
Ag & 0.33 \\
Au & 0.35 \\
Cu & 0.30 \\
Diamond & 0.83 \\
Ge & 0.76 \\
Si & 0.57 \\
GaAs & 0.55 \\
GaSb & 0.56 \\
Insb & 0.50 \\
Zns & 0.43 \\
\hline
\end{tabular}




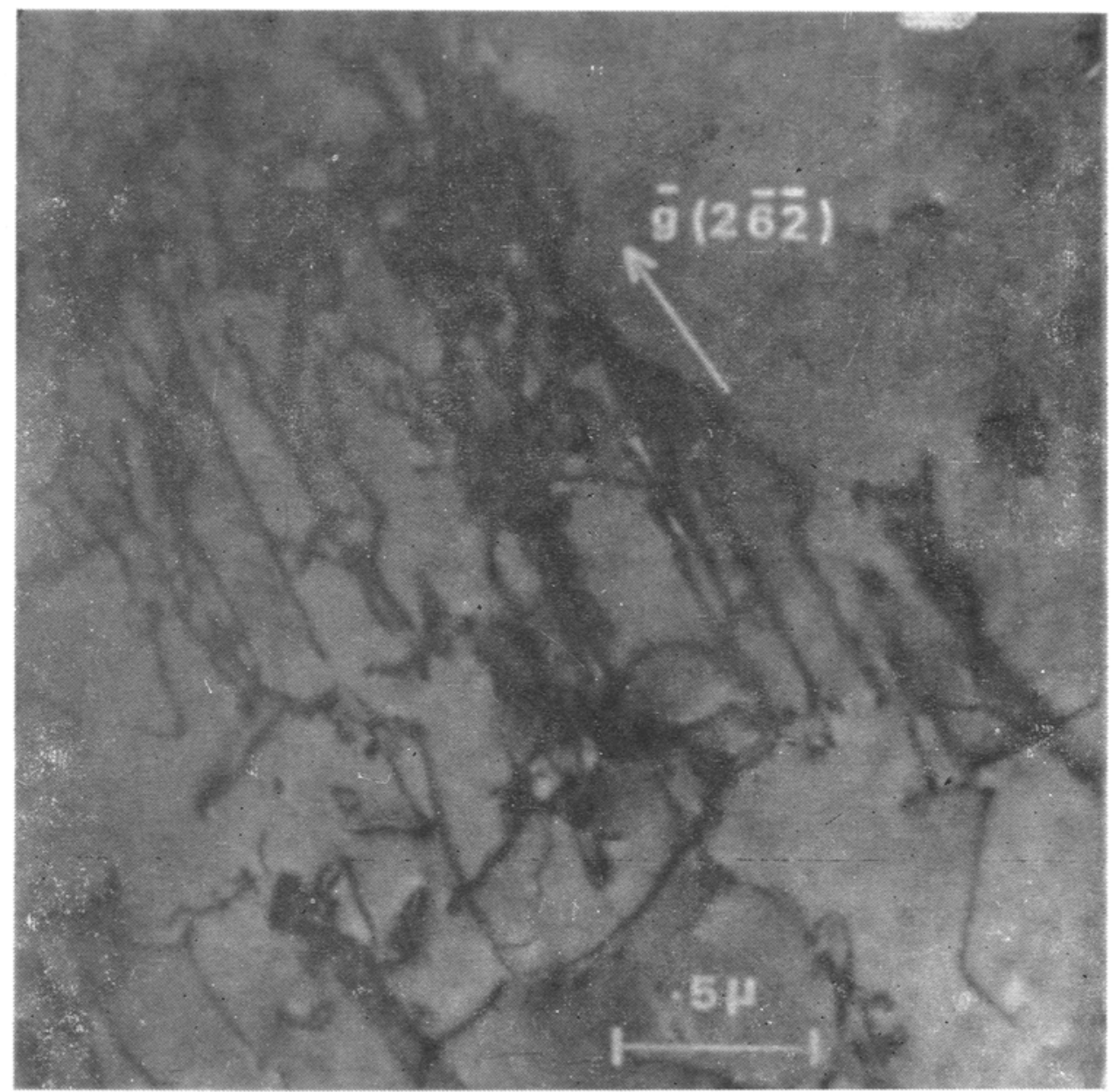

Figure 9. Dislocation substructure of Si-doped GaAs deformed at $550^{\circ} \mathrm{C}$ in compression to a $3 \%$ strain (Swaminathan 1975). 

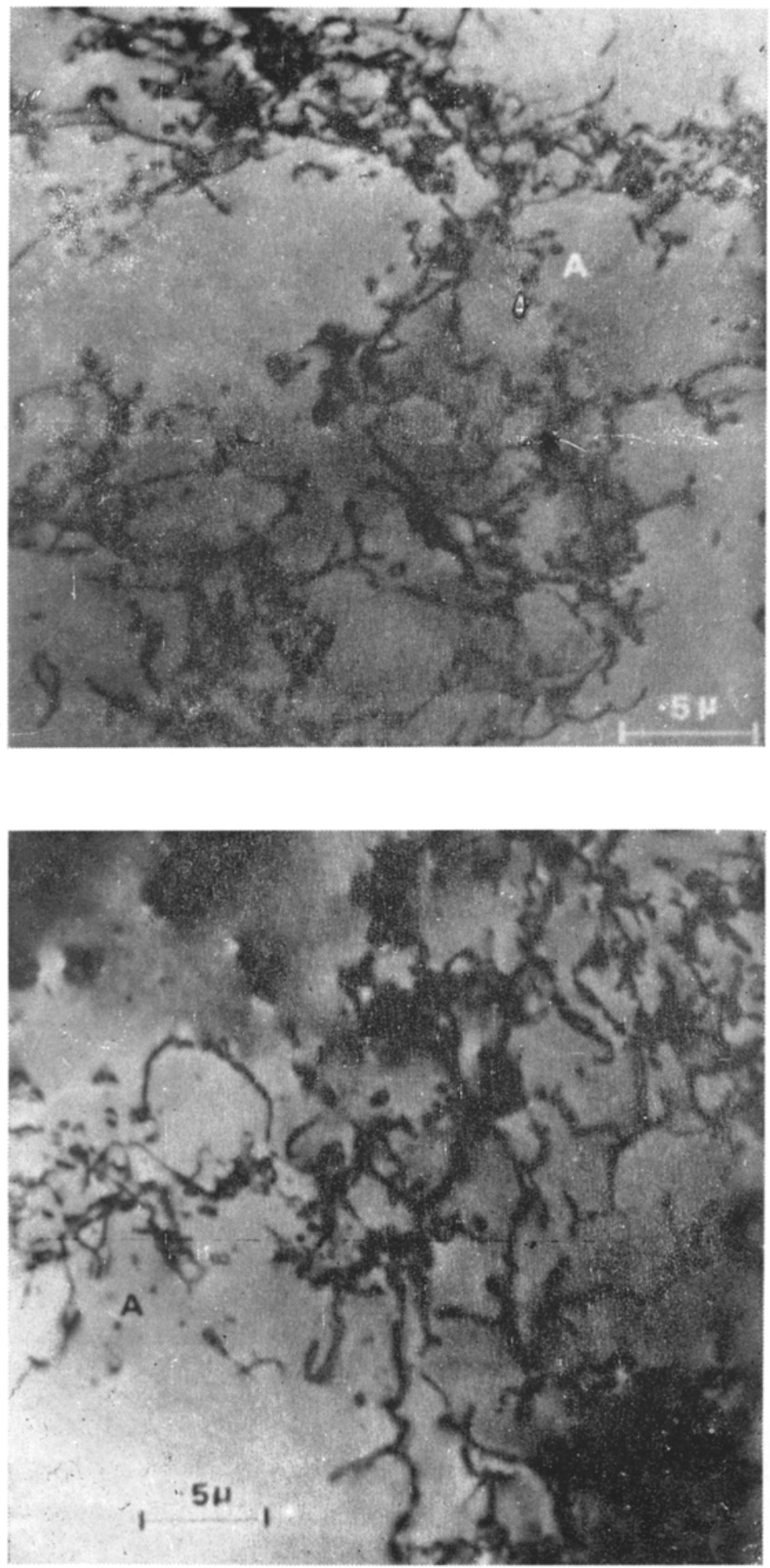

Figure 10. Dislocation su'sstructures of Si-doped $\mathrm{GaAs}$ deformed at $550^{\circ} \mathrm{C}$ in compression to a $20 \%$ strain (Swaminathan 1975 ). 
1953). However, in the case of $\alpha B$ planes, since glide takes place between closely spaced planes dissociation into partial dislocations can readily occur, which is not the case for dislocations in Aa planes as we shall see below.

\subsection{Partial dislocation and stacking faults}

A stacking fault occurs in the zinc blende structure in the same way as it does in fcc lattices. If the $A \alpha B \beta C \gamma$ stacking sequence of $\{111\}$ planes shown in figure $6 \mathrm{c}$ is written as simply $A B C A B C$ then the insertion of a $B$ plane results in $A B C B A B C$ ... i.e., an extrinsic stacking fault and the removal of a $B$ plane results in $A C A B C$ i.e., an intrinsic fault. Because of the stacking of planes in pairs, a low energy fault is formed only between the $\alpha B$ planes (glide set) (Hirth and Lothe 1968). Where a stacking fault ends, a partial dislocation occurs. The partials associated with a $60^{\circ}$ dislocation are the analogues of the Shockley partials in fec lattice; i.e., they have Burgers vectors $a / 6$ [211] and $a / 6$ [151].

With the development of weak beam diffraction contrast analysis in the transmission electron microscope (Cockayne et al 1969) it has now been established that the dislocations in III-V compounds (Gottschalk et al 1978) are dissociated into Shockley partials. In GaAs (Gomez and Hirsch 1978) both $\alpha$ and $\beta$ dislocations were found to be dissociated and to have the same separation. Even in alloy semiconductors such as $\mathrm{Ga}(\mathrm{As}, \mathrm{P})$ the dissociation was observed (Mader and Blakeslee 1974). (From the separation of the partials the intrinsic stacking fault energy in undoped $n$-type GaAs $\left(n \sim 2 \times 10^{16} \mathrm{~cm}^{-3}\right)$ was estimated to be $48 \pm 6 \mathrm{erg}$ $\mathrm{cm}^{-2}$ (Hirsch 1979a). The stacking fault energy was found to be lower in Te-doped $\left(n \sim 10^{18} \mathrm{~cm}^{-3}\right)$ and Sn-doped $\left(n \sim 10^{16}-10^{17} \mathrm{~cm}^{-3}\right)$ GaAs (Astakhov et al 1980) indicating the influence of impurities on the dissociation of dislocations. The observation that dislocations in $\mathrm{GaAs}$ as well as in other semiconductors are dissociated raises doubt about the so far accepted concept that dislocations glide in the Aa planes (shuffle set) since dissociation in these planes produces a high energy fault. The conflict between mobility and dissociation may be reconciled by a model proposed by Hornstra (1958) for the dislocation in the shuffle set. The idea is that the mobile shuffe set dislocation might associate itself on coming to rest with a pair of partials of opposite sign on the neighbouring glide set planes. The corresponding reaction is

$$
\begin{aligned}
\frac{a}{2}[110]+\frac{a}{6}[\overline{12} \overline{1}]+\frac{a}{6}[\overline{12} 1] & =a / 6[2 \overline{1} \overline{1}] \\
+ & \frac{a}{6}[\overline{12} 1]
\end{aligned}
$$

Hirth and Lothe (1968) proposed that a dislocation in the glide set can transform to one in the shuffle set and vice versa by acquiring a row of either interstitials or vacancies; that is, the process occurs by climb. The energetics of the vacancy. and interstitial type shuffe set dislocations in the diamond lattice has been studied by Blanc (1975). The motion of the partial dislocations created in this manner involves shuffling of the bonds and hence is the name shuffle set. 
It has been, however, not possible to decide between the glide and shuffle sets by means of electron microscopy since the strain field of the partial dislocations created in the above manner and by the straight dissociation of the glide set dislocation is practically the same. Recently, Hirsch (1981) reviewed this aspect in detail and suggested that the glide set configuration should be favoured. Further experimental and theoretical work are neccessary before this question can be settled unambiguously.

\subsection{Electrical properties of dislocations}

The early pioneering work of Shockley (1953) and Read (1954) on dislocations in diamond cubic structure showed that a $60^{\circ}$ dislocation has a row of atoms each with an unpaired bond (dangling bond) along the edge of the extra half plane. For dislocations in the $\{111\}$ planes the line density $(d)$ of such bonds is equal to $(2 / \sqrt{3 b}) \sin \theta$ where $\theta$ is the angle between the dislocation line and the Burgers vector $b$. Accordingly, the screw dislocation does not have any dangling bond. The presence of these dangling bonds form the most important feature of dislocations in semiconductors determining to a large extent the electrical and the optical properties of dislocations.

The unsaturated dangling bond along the dislocation in $\mathrm{Si}$ or $\mathrm{Ge}$ can either accept another electron to satisfy the bond or donate its electron and in this sense the dislocation can act as an acceptor or a donor. Because of the translational symmetry along the dislocation it is supposed to form an one-dimensional band (Shockley 1953), the band width and the mobility in the band being determined by the overlap of wave functions of electrons on neighbouring atoms along the dislocation. In GaAs, because of the different core structure of the $\alpha$ and $\beta$ dislocations they would be expected to have different electrical characteristics.

The electrical behaviour of dislocations in III-V compounds has been studied in detail only for InSb. Specimens which were bent to introduce a surplus of In- or Sb-dislocations exhibited differences between the two types of dislocations. In n-type InSb Gatos et al (1961) found that the In-dislocations act as acceptors while Sb dislocations act as donors. Bell et al (1966) on the other hand reported acceptor action for both dislocations in $n$-type $\mathrm{InSb}$ and donor action for $\mathrm{Sb}$ dislocations in $p$-type InSb. In $p$-type InSb Baitinger et al (1969) found only an acceptor action for $\mathrm{Sb}$ dislocations but both acceptor and donor behaviour for In dislocations. Recently Labusch and Schröter (1980) suggested that a possible reason for these conflicting results could be the difficulty in identifying whether the dislocation in question belongs to the shuffle set or the glide set. This is important because as we noted earlier an In dislocation in the glide set is a Sb-dislocation in the shuffle set and vice versa.

Information about the electronic states of dislocations in GaAs are very scarce. The electrical properties of dislocations are generally deduced from measurements made on deformed samples. From Hall measurements on $\mathrm{Cr}$-doped high resistivity $\mathrm{GaAs}$ bent at $580^{\circ} \mathrm{C}$ to produce an excess of $\alpha$-dislocations, Lin and Bube (1975) observed an increase in the electron concentration after deformation. On the other hand, Esquivel et al (1976) observed a decrease in carrier concentration and mobility 
in $n$-type Te-doped and undoped $\mathrm{GaAs}$ which has been previously bent to introduce an excess of either $\alpha$ or $\beta$-dislocations with the decrease being larger in samples containing $\beta$ dislocations. Similar observations were made by Nakata and Ninomiya (1977) in $n$-type GaAs and by Gwinner and Labusch (1979) in both $n$ and $p$ type undoped GaAs with a difference namely that the sample with $\alpha$-dislocations showed a larger decrease in the carrier concentration (Gwinner and Labusch 1979). These results suggest acceptor and donor action for the dislocations in $n$ - and $p$-type samples respectively. From analyzing spectral response and decay process of photoconductivity in bent undoped n-type GaAs, Nakata and Ninomiya (1979) deduced two dislocation levels, one at $0.7 \mathrm{eV}$ and another at $0.9 \mathrm{eV}$ below the conduction band edge and they were attributed to two partial dislocations. Deep level transient spectroscopic measurements (Ishida et al 1980) on undoped $n$-type GaAs which has been deformed under compression indicated no electron trap but a broad unresolved hole trap spectrum presumably due to overlapping dislocations of different character.

Theoretical calculations by Farvacque and Ferre (1980) predicted deep levels for both $\alpha$ and $\beta$ dislocations in GaAs. Oberg (1978) using a Slater-Koster approach found that the $60^{\circ} \mathrm{Ga}$ and As dislocations act as acceptors in $n$-type and the As-dislocations act as donors in $p$-type GaAs. Both these calculations were made for perfect dislocations belonging to the shuffle set. This may be unrealistic in view of the experimental evidence that dislocations in GaAs are dissociated. Further the uncertainty whether a particular dislocation observed in an experiment is the glide type or shuffle type is another problem in comparing experiment and theory.

Finally there are two other points worth mentioning in the discussion on the electrical properties of dislocations. Firstly, the electrical nature of the dislocation is a natural consequence of the presence of unsaturated 'dangling' bonds along the dislocation. This picture holds good even for the screw dislocations in light of the new evidence from EPR measurements on deformed Si which indicates that even they contain dangling bonds (Alexander 1979). It is, however, possible that the dangling bonds at the end of the dislocation interact and get eliminated as a result. For example, the $90^{\circ}$ edge dislocation in $\{100\}$ plane has the two dangling bonds per atom which can undergo reconstruction eliminating dangling bonds (Hornstra 1958). Further, calculations by Marklund (1979) and Jones (1979) on partial dislocations in Si and Ge suggest that reconstruction should be energetically favoured. In fact, such a reconstruction was suggested to be the reason for the absence of carrier recombination at an edge sessile dislocation in (Ga, Al) (As, P) heterostructures (Petroff et al 1980). This may also be the reason why no dislocation induced luminescence was observed in deformed GaAs (Bohm and Gwinner 1978).

Secondly, some of the electrical effects in deformed GaAs such as the decrease in the carrier concentration and mobility could very well be due to point defects. For example, at the temperature of deformation, impurities in GaAs could diffuse and precipitate near dislocations and thus the changes in resistivity would merely be a result of this gettering near dislocations. This also emphasizes the need for high purity GaAs samples for the experiment. Another possibility is the creation of a high density of point defects during deformation due to 
dragging jogs on screw dislocations as shown for example in $\mathrm{Si}$ (Weber and Alexander 1977 and Kimerling and Patel 1979) and Ge (Albers et al 1977). Ishida et al (1980) found in a DLTS study on deformed GaAs that two electron traps and a hole trap which were present in the as-grown material increased with plastic deformation. These may very well be related to point defects. Since there could be a concentration of these point defects near the dislocation a simple interpretation of the electrical results in deformed GaAs may be incorrect.

\subsection{Mechanical properties of GaAs and dislocations}

The addition of dopants has a profound influence on the dislocation velocity and thus the mechanical properties of GaAs. Several investigators (Sazhin et al 1966, Laister and Jenkins 1973 and Swaminathan and Copley 1975a) have noted that addition of $n$-type dopants like $\mathrm{Si}, \mathrm{Te}$ increases the yield stress while that of $p$-type dopants like $\mathrm{Zn}$ decreases the yield stress. This effect was confirmed in dislocation velocity measurements as well (Choi et al 1977). Though there were differences in the effects of doping on the different types of dislocations, in general the velocity of the dislocation was decreased with $n$-type doping and increased with $p$-type doping consistent with the yield stress measurements.

To explain the effect of a small amount of impurity (typically around $10^{18} \mathrm{~cm}^{-3}$ ) it has been proposed (Sazhin et al 1966 ; see also Kirkby 1975) that dislocations are pinned by impurity atmospheres. Swaminathan and Copley (1975a) argued against the pinning model because of their observation that yielding was gradual and not abrupt, the latter phenomenon being characteristic of dislocation pinning. In view of the small concentration of impurities, Swaminathan and Copley (1975a) proposed that hardening in $n$-type GaAs occurs by the interaction of mobile dislocations with asymmetrical defects such as donor-vacancy impurity complexes. Swaminathan and Copley (1975b) investigated in detail the effect of various thermal and thermomechanical treatments on the yield stress of heavily Si-doped GaAs and developed a point-defect model to explain the observed behaviour in terms of changes in the concentration of $\left(\mathrm{Si}_{\mathrm{Ga}}-\mathrm{V}_{\mathrm{Ga}}\right)$ pairs.

Alternate theories have been proposed especially in $\mathrm{Si}$ and $\mathrm{Ge}$ to explain the effects of doping on dislocation velocity (Haasen 1975; Patel et al 1976 ; Schröter et al 1977; Patel and Testardi 1977) based on the influence of doping on the line charge of the dislocation and thus on the formation and/or motion of double kinks on the dislocation. Recently Hirsch (1979b) proposed another model where he assumed localized electronic states associated with kinks and the doping effect was considered to be due to the change in the charge states of the kinks themselves. Though such electronic effects may be important in doped GaAs as well especially at low deformation temperatures, a comprehensive theory taking into account the elastic/electronic interaction between dislocations and asymmetrical impurity complexes is needed to understand the dislocation motion. Such a study is also important from a practical point of view in that a major failure mechanism of GaAs-(Al, $\mathrm{Ga}$ )As heterostructure lasers has been attributed to the motion of dislocation networks known as dark line defects (\$4). 


\subsection{Control of dislocation density in GaAs}

Since dislocations generally cause deleterious effects in devices $(\S 4)$, it is aimed to grow dislocation free material as far as possible. Dislocations in the growing crystal can be introduced in a variety of ways:

(i) Thermal stresses causing plastic deformation.

(ii) Condensation of the excessive point defects present near the growth temperature to form prismatic dislocation loops.

(iii) Defective seed crystal or accidental introduction of macroscopic foreign particles during growth causing generation and multiplication of dislocations.

Of these three, the first two are the most important aspects to be considered. Brice and King (1966) and Brice (1970) noted that the partial pressure of As during growth is a parameter for controlling the dislocation density in horizontal Bridgman crystals and pulled crystals. A low partial pressure of As was recommended so as to reduce the concentration of $V_{\mathrm{Ga}}$ and thus the concentration of prismatic loops.

Perhaps the principal mechanism by which dislocations are incorporated in the crystal is the plastic deformation caused by excessive thermal stress (Osvenskii and Eremeev 1971 ; see also Jordan et al 1980 and references therein). A detailed analysis of this problem in GaAs is to be found in the paper by Jordan et al (1980). They concluded that dislocations in pulled crystals cannot totally be avoided unless $h \approx 0.02 \mathrm{~cm}^{-1}$ for $\triangle T \sim 200^{\circ} \mathrm{C}$ where $h$ is the heat transfer coefficient and $\triangle T$ is the temperature difference between the melting point of $\mathrm{GaAs}$ and the ambient temperature. In this regard these authors suggested the incorporation of radiation shields and an after-heater in the growth system.

Since the dislocation generation in the as-grown crystals is caused by plastic deformation, dopants which increase the yield stress would tend to lower the dislocation density. We noted in $\S 3.4$ that the yield stress increases by the addition of donors and decreases with acceptors. Addition of donor impurities giving carriers $1-8 \times 10^{18} \mathrm{~cm}^{-3}$ led to a substantial reduction in the dislocation density while addition of acceptor impurities even up to the solubility did not have any effect (Osvenskii and Eremeev 1971 and Belyatskaya et al 1972). The addition of donors also resulted in a significant rearrangement of the dislocations. This is presumably because of the high vacancy concentration in doped crystals facilitating dislocation climb. In fact Swaminathan and Copley (1975b) found that the microstructure of $\mathrm{Si}$-doped GaAs sample which has previously been annealed to produce a high density of prismatic dislocation loops was found to be free from loops and glide dislocations after deformation to a few per cent strain and recovery at $400^{\circ} \mathrm{C}$ for a few hours.

\section{Effects of native point defects and dislocations on devices}

It is now generally accepted that native defects and dislocations have profound influences in determining the quality and performance of semiconductor devices. 
However, the correlation between device characteristics and defects has remained qualitative. The reason for this is that it is often difficult to isolate pure dislocation effects when possibility of dislocation-point defect interaction exists. In the case of native defects it has been even more difficult to correlate device parameters with any specific native defect. The implication of such defects is often based on circumstantial evidence. For example, the thermally damaged layer present on semi-insulating $\mathrm{GaAs}$ substrates during the growth of epitaxial films has been found to have adverse effects on the performance of microwave FET devices (Barrera 1975). The problem has been ascribed to $V_{\mathrm{As}}$ or impurity $-V_{\mathrm{As}}$ complexes (Lum et al 1977). This is a natural assignment since during baking of the substrates in an open system at high temperatures prior to epitaxial growth, As escapes from the crystal giving rise to $V_{\mathrm{As}}$. The $V_{\mathrm{As}}$ related defects have been thought to be the cause of the $p$-layer formation on the substrates. Recently, Klein et al (1980) showed that $\mathrm{Mn}$ present in the $\mathrm{Cr}$-doped substrates diffused to the surface during heat treatment. Since $\mathrm{Mn}$ is an acceptor in GaAs, the type conversion could very well have been caused by it.

Ettenberg (1976) found in vapour grown GaAs that both the minority carrier diffusion length and life time decrease with increasing $\mathrm{As} / \mathrm{Ga}$ ratio during growth. Since the formation of $V_{\mathrm{Ga}}$ is favoured-with increasing As/Ga ratio, the observed effects are attributed to it. A similar implication was made by Kasano (1978b) for degradation of vapour grown GaAs. Again both these effects could have been caused by some impurity or some other $\mathrm{Ga}$ deficient nonstoichiometric defect.

There is perhaps less ambiguity about the effects of dislocations. (For some earlier references see the review by Petroff 1975). Dislocations reduce the minority carrier diffusion length and when it is comparable to the spacing between them the luminescence efficiency decreases (Ettenberg 1974). High resolution

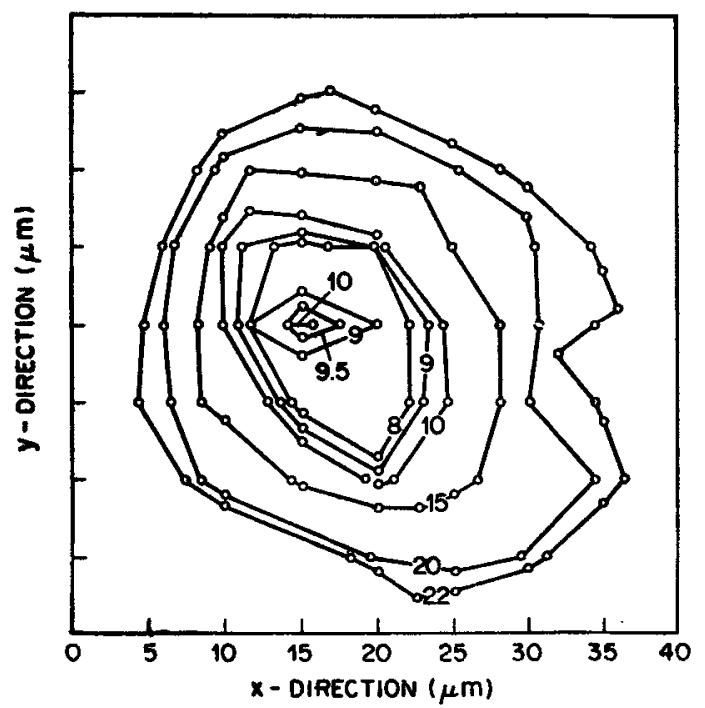

Figure 11. Typical isointensity luminescence map in the vicinity of a dislocation in GaAs. The numbers indicate the intensity in arbitrary units for the near gap luminescence at $1.513 \mathrm{eV}$ at helium temperatures (Heinke and Queisser 1974). 

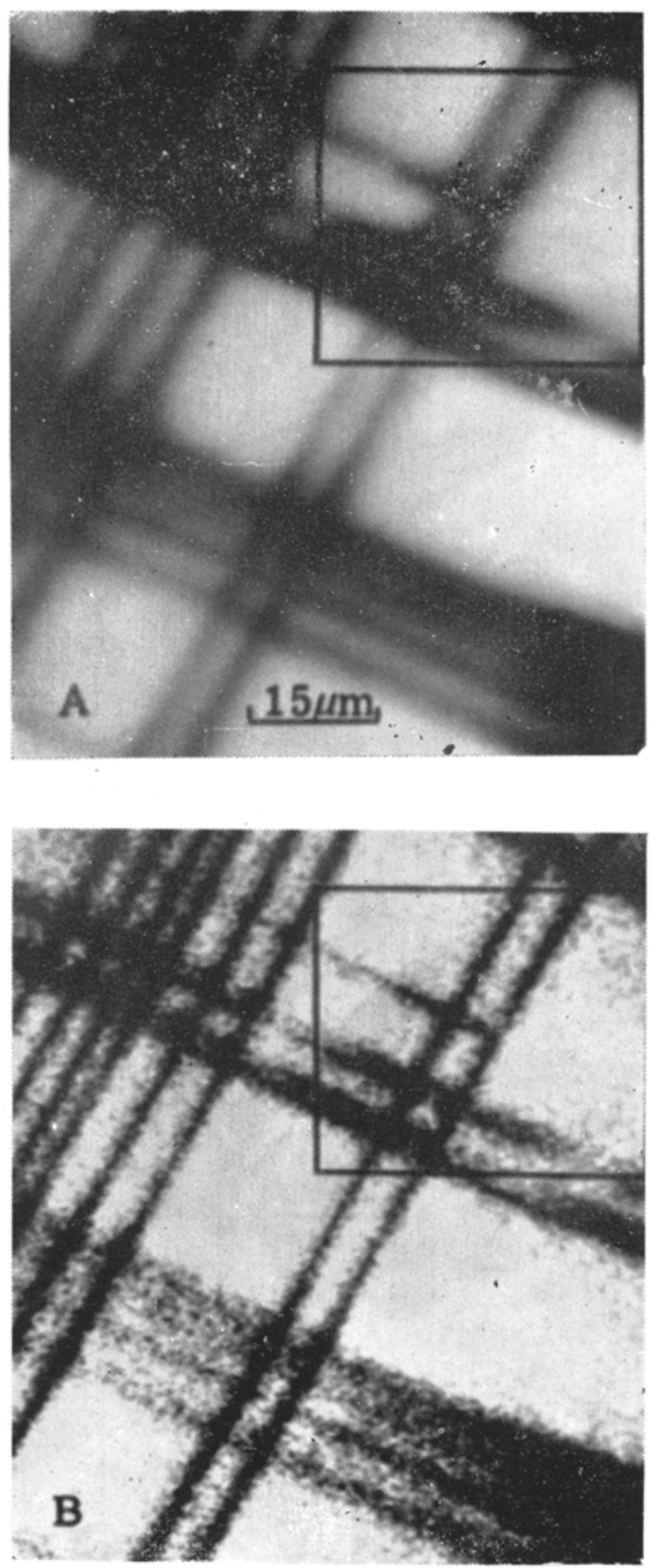

Figure 12 A, B. see page 432 for caption 

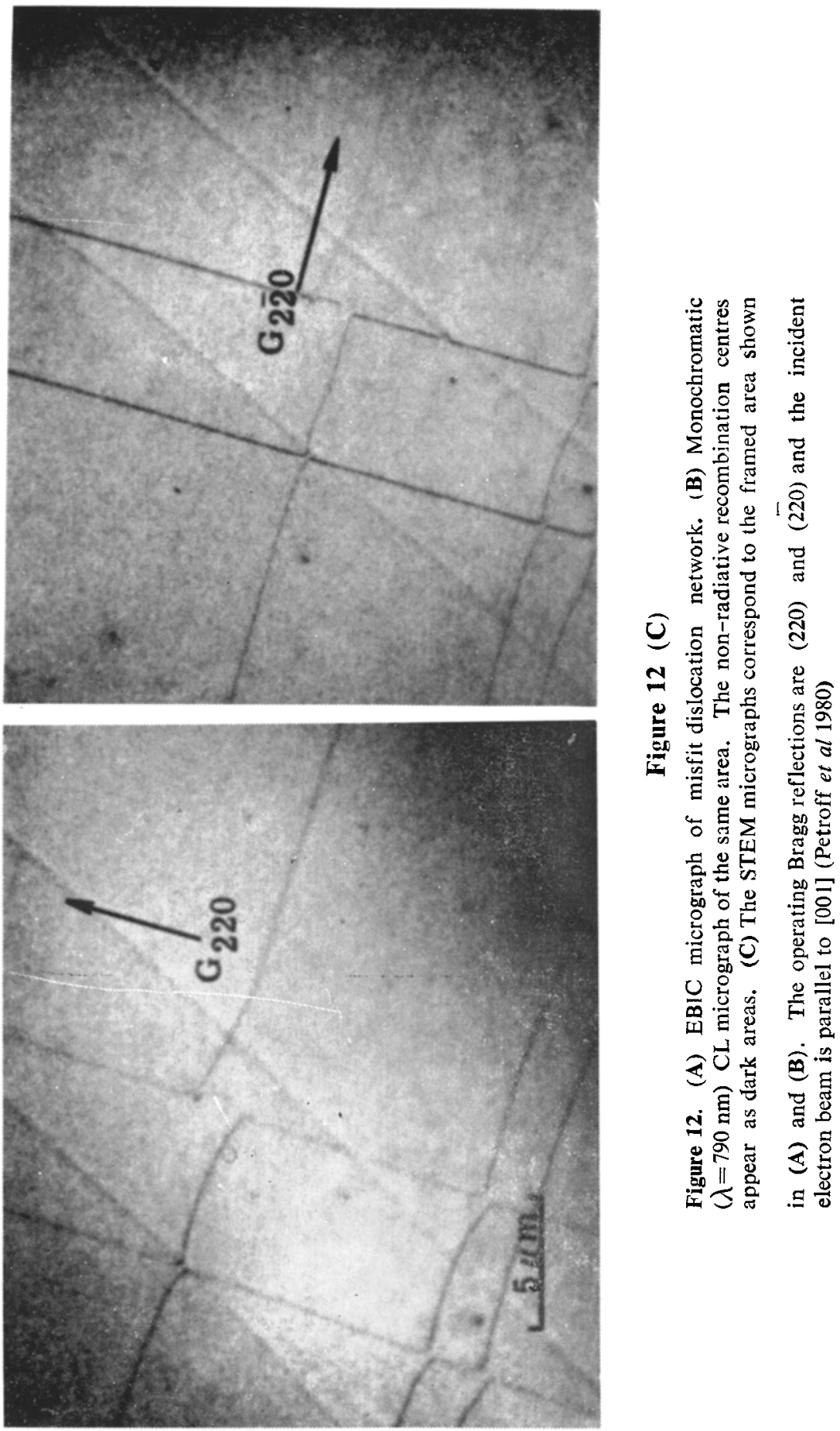
photoluminescence scanning around dislocations at low temperatures (Heinke and Queisser 1974) showed that the intensity of band edge emission decreases near a dislocation within a cylinder of $20 \mu \mathrm{m}$ radius around it (figure 11). The authors also noted that the effect is greater near freshly introduced dislocations than grown-in dislocations by nearly three orders of magnitude. A similar observation was also made earlier by Osvenskii et al (1971). However, Böhm and Fischer (1979) disputed this result and noted that both grown-in and deformation induced dislocations behave similarly. Further they made a significant observation that the larger effect of deformation induced dislocations as noted by Heinke and Queisser (1974) was indeed due to $\mathrm{Cu}$ segregating near dislocations. The importance of such impurity-dislocation interaction was also evidenced in the results of Vovnenko et al (1979) who reported an increase in the internal quantum efficiency of some deep centre luminescence in deformed GaAs. Though such effects could mask dislocation effects it is generally true that dislocations act as nonradiative centres. This is clearly shown in figure 12 for dislocations in $\mathrm{Ga}_{1-x} \mathrm{Al}_{x} \mathrm{As}_{1-y} \mathrm{P}_{y}$ heterostructures grown by LPE. Dislocations labelled $D_{2}, D_{3}$ and $D_{4}$ act as nonradiative centres giving dark contrast. Dislocation $D_{1}$ which does not exhibit any contrast has been assigned to a neutral edge dislocation.

That dislocations act as nonradiative centres is further shown by Roedel et al (1979) who investigated the effect of dislocations in graded bandgap Si-doped $\mathrm{Ga}_{1-x} \mathrm{Al}_{x}$ As light emitting diodes. This is shown in figure 13. The external quantum efficiency of the diodes is found to decrease with increasing dislocation density in the LPE wafers.

Dislocations are also known to be the cause of the rapid degradation of $\mathrm{Ga}_{1-x} \mathrm{Al}_{x}$ As - GaAs injection lasers and LEDs. The degradation has been linked to linear defects known as dark line defects (DLDs). The DLDs have been

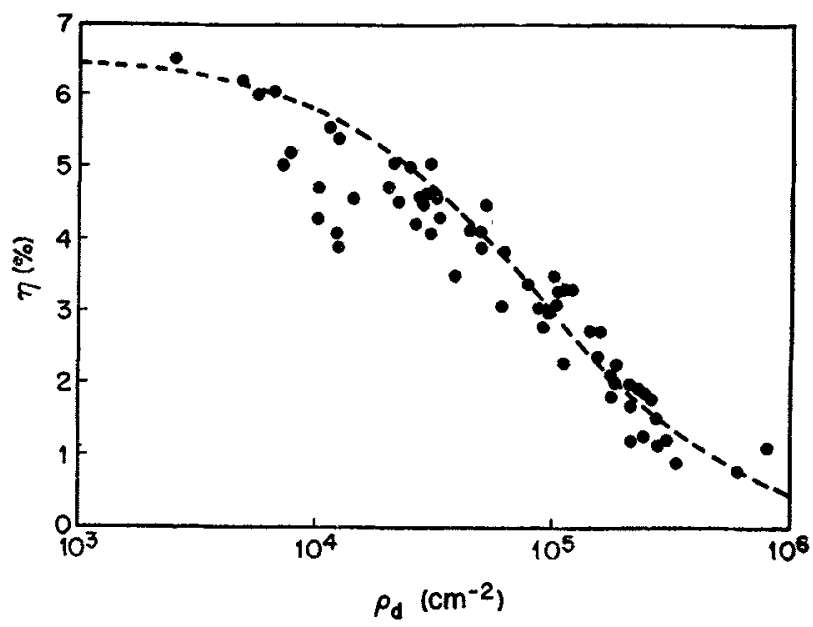

Figure 13. A plot of the efficiency $v$ dislocation density for 45 individual LEDs and the results of the substrate mapping experiment and the theoretical approximation for $\eta(\rho)$ dashed line (Roedeal et al 1979). 


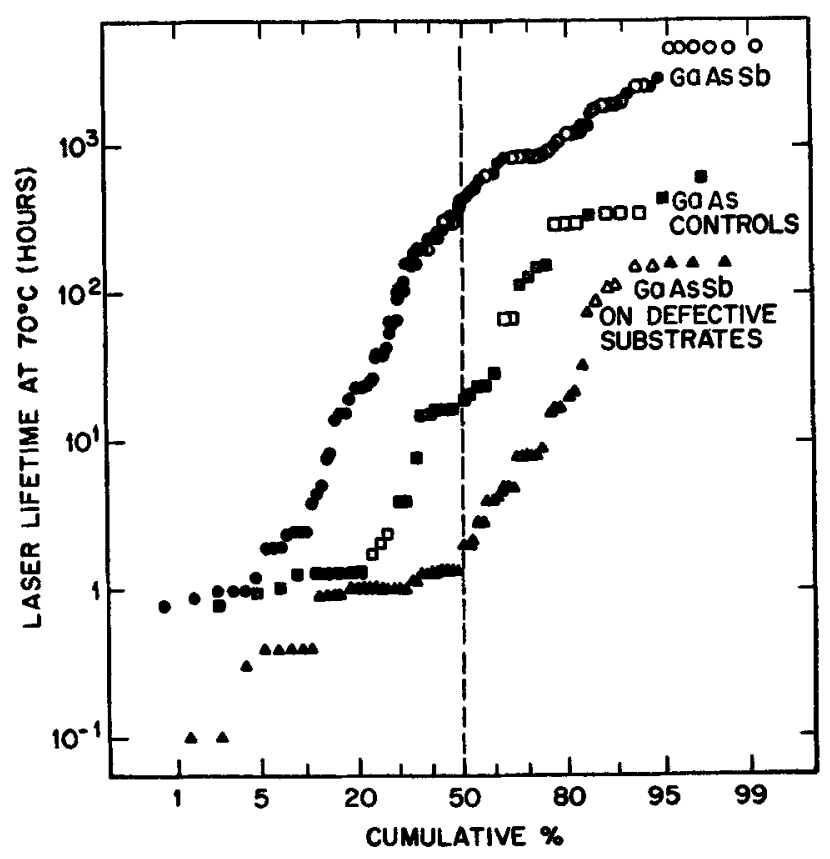

Figure 14. Plot of the time to failure (filled symbols) or the accumulated testing time (open symbols) for lasers operated at $3 \mathrm{~mW}$ at $70^{\circ} \mathrm{C} v s$ the cumulative percent of devices with times less than or equal to that time, on a normal scale. The Ga (As, Sb) devices have GaSb concentrations of between 0.4 and 2 mole \% while the GaAs controls have $\mathrm{GaSb}$ concentrations of zero or less than 0.1 mole \%. These were grown on substrates which had dislocation densities of $<3 \times 10^{3} \mathrm{~cm}-2$. The (GaAs) Sb lasers grown on defective substrates had substrates with dislocation densities of $\sim 4 \times 10^{4} \mathrm{~cm}^{-2}$. Not all devices were monitored at fraction of hour intervals for times less than one hour (Anthony et al 1981).

identified by transmission electron microscopy as three dimensional dislocation networks consisting of long dislocation dipoles and small dislocation loops. The DLDs originate from a precurors defect known as dark spot defects (DSDs) which themselves nucleate from threading dislocations in the device active layer. Figure 14 compares the lifetime at $70^{\circ} \mathrm{C}$ of two classes of $\mathrm{Ga}_{1-x} \mathrm{Al}_{x}$ As injection lasers having $\mathrm{Ga}(\mathrm{As}, \mathrm{Sb})$ active layers. The devices which have poor $70^{\circ} \mathrm{C}$ median lifetimes were made from LPE wafers which were grown on substrates having a dislocation density of $\sim 4 \times 10^{4} \mathrm{~cm}^{-2}$ as compared to the good devices for which the substrates had dislocation densities $<3 \times 10^{3} \mathrm{~cm}^{-2}$ (Anthony et al 1982). The probability of a dislocation threading through the active layer and hence that of DLD formation increase with increasing number of dislocations in the substrates and thereby affecting the laser operating lifetime.

The growth of the DLDs which may nucleate at the threading dislocations occurs very rapidly $\left(10^{-6}-10^{-2} \mathrm{~cm} / \mathrm{sec}\right.$ ) (Petroff and Hartman 1974; Monemar and Woolhouse 1976) and the growth rate is dependent on the injection current of the laser. The DLDs propagate usually in $\langle 100\rangle$ direction and occasionally in 
$\langle 110\rangle$ direction. While the DLD in the $\langle 110\rangle$ direction has been shown to be caused by carrier injection enhanced (Kimerling and Lang 1975) dislocation glide (Kamejima et al 1977) there is controversy about the $\langle 100\rangle$ DLD. A pure climb process involving either the absorption of interstitials or the emission of vacancies has been invoked to explain the observed $\langle 100\rangle$ DLD structure (Petroff and Hartman 1974 and Hutchinson and Dobson 1975b). On the other hand, Matsui et al (1975) proposed a glide mechanism for the initial stage of the DLD growth in the $\langle 100\rangle$ direction and a combination of glide and climb for the final development of the observed structure. The important point that emerges from these models is that climb plays an important role in DLD growth. There is, however, considerable uncertainty about the nature and origin of the point defects required for climb. We will refer the reader to the book on heterostructure lasers by Casey and Panish (1978) for a discussion of these topics. We will discuss only a few recent works here.

Hutchinson et al (1978) investigated the dislocation structures in annealed Tedoped GaAs after optical pumping. The annealing treatment was such that it produced in the samples prismatic dislocation loops which were assigned to interstitials based on transmission electron microscopic contrast analysis. A $1 \mu_{\mathrm{m}}$ layer of $\mathrm{Ga}_{0.7} \mathrm{Al}_{0.3}$ As was grown by LPE on the annealed GaAs sample which was then optically pumped with a 7510A line of a $\mathrm{Kr}$ laser at the power density of $5.7-7.9 \times 10^{4} \mathrm{~W} / \mathrm{cm}^{2}$ for times varying from 1 to $80 \mathrm{~min}$. The pumped regions were investigated by transmission electron microscope. Hutchinson et al observed that optical pumping caused the loops to undergo considerable climb and develop into long dislocation dipoles. The extent of the climb was found to increase with pump power and time. Since the presence of the prismatic interstitial dislocation loops in the sample which has been equilibrated prior to optical pumping implied that the concentration of free interstitials in solution is very small, it was concluded that climb had occurred by vacancy emission from the dislocation. The latter process was supposed to have been caused by the energy released in the recombination process (O'Hara et al 1977). Hutchinson et al 1978 proposed that the vacancies emitted in this process are not observable by transmission electron microscope as they might aggregate to form submicroscopic clusters. The major difficulty in accepting this model unequivocally is the controversy regarding interstitial vs. vacancy dislocation prismatic loop in annealed GaAs (see § 2.4)

Wakefield (1979) examined the effect of strain on the photoluminescence degradation in oxide striped $(\mathrm{Ga}, \mathrm{Al})$ As double heterostructure lasers and found that dark bands were observed in regions where the dilation was a maximum. The photoluminescence degradation was attributed to recombination enhanced migration of point defects to the strained region. Since regions of tension were degraded, the point defects were suggested to be interstitial atoms. This conclusion is in agreement with the viewpoint that DLD climb occurs by the absorption of (and concomitant emission of) interstitials (vacancies).

Using a scanning deep level transient spectroscopy technique Lang et al (1979b) investigated the interaction between dislocation climb networks and the so-called DX centres (see § 2.4) in $(\mathrm{Ga}, \mathrm{Al})$ As heterostructures. These authors found that near the dislocation climb network the concentration of the DX centres decreased by $\sim 40 \%$ (figure 15 ). This suggested that there is an interaction 


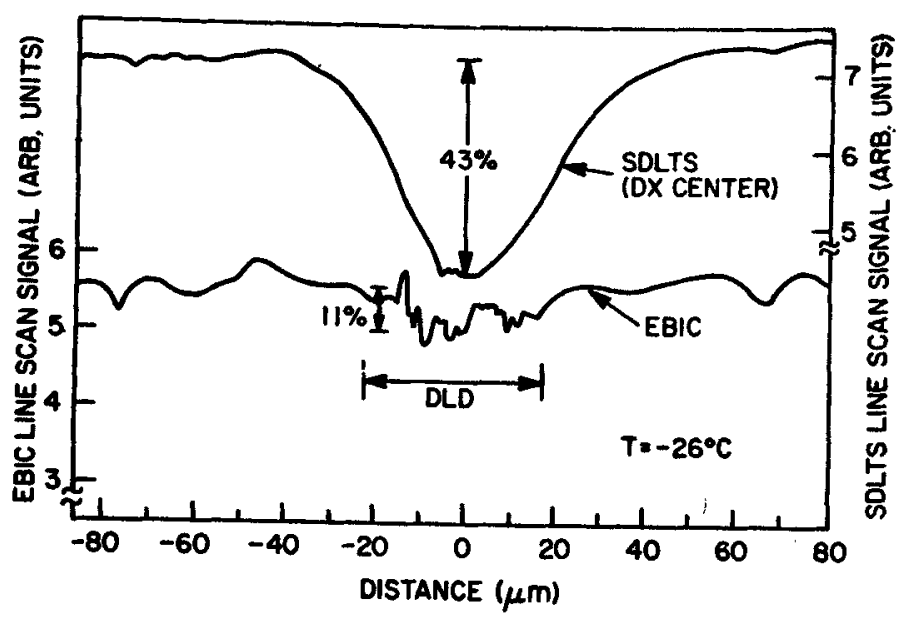

Figure 15. Steady-state current (EBIC) and transient-detrapping-current (SDLTS) intensities as a function of the beam position for a beam scanning across a $\langle 100\rangle$ dark line (DLD). The specimen temperature is $\mathrm{T}=-26^{\circ} \mathrm{C}$ to display the DX-centre distribution with the SDLTS line scan (Lang et al 1979).

between the DX centres and the dislocation network. It is not, however, clear whether the DX centres are themselves the source of the point defects involved in the climb process because of the apparent conflict between the proposed vacancy nature of the DX centre (Lang et al 1979a) and interstitial nature of the dislocation network. To resolve this contradiction Lang et al hypothesized that the change in the DX centre occurred before the climb process and that the decay of the centre during electron-hole recombination might proceed by the emission of $\mathrm{Ga}_{i}$. Recently, Frank and Gösele (1980) reiterated the earlier model proposed by Petroff and Kimerling (1976) that dislocation climb occurs by absorption of $\mathrm{Ga}_{i}$ and speculated that the DX centres might in fact be $\mathrm{Ga}_{i}$.

Figielski (1980) made a new proposal to explain the degradation of lasers. He suggested that during carrier injection bond breaking and reconstruction occurs. This process originates at a seed dislocation. During such reconstruction, migration of the broken dangling bond occurs leaving behind a line defect. The line defect thus created may be screw or a helical dislocation. Figielski further suggested that the dislocation dipoles could develop by interstitial pipe diffusion along the helical dislocations. This model according to Figielski explains at least the highly directional nature of dislocation dipoles, the high velocity of dipole propagation, unusual dipole configuration and shape and the role of carrier injection in the process. It may be interesting to check this hypothesis by opticaly pumping GaAs substrate material which contains helical dislocations introduced by a suitable previous annealing treatment. 
Hutchinson and Dobson (1980) reported an asymmetry in the climb rate, that is, the $\alpha$ dislocations (shuffle set) climb much more rapidly than the $\beta$ dislocations (shuffle set) indicating that the climb process depends on the structure of the dislocation core. This might play an important role in the point defect-dislocation interaction necessary for the climb. Imai et al (1979) also suggested that the fast and slow DLDs might be dominated by $\sim \alpha$ and $\beta$ dislocations respectively.

We will conclude this section with an observation by Petroff (1981) that the nature of the DLD structure in lasers grown by liquid phase epitaxy or molecular beam epitaxy are identical. Petroff suggested that the inhomogeneity in the interfaces of the LPE structures and point defects intrinsic to the MBE structures could be one of the likely sources of point defects for DLD growth. Petroff further noted that dislocations in multiquantum well superlattices are not nonradiative centres. This finding suggests the possibility of making more reliable lasers with such structures.

\section{Conclusions}

We have given a comprehensive review of defects in GaAs with emphasis on native point defects and dislocations. The current state of knowledge on point defects does not allow us to draw a unified defect model for several reasons. First, the question of the dominant native defects in equilibrium is unsettled. This is important both from a fundamental and technological point of view. Hurle (1979) proposed the Frenkel disorder in the As-sublattice as the dominant disorder in equilibrum. Recent EPR result of Wagner et al (1980) suggests the presence of $\mathrm{As}_{\mathrm{G}}$ defect. Further the acceptors observed after heat treatment at low As pressure could be assigned to $\mathrm{Ga}_{A s}$ instead of $V_{\text {As }}$ as had been done. The $A s_{G_{a}}$ defects could perhaps also be invoked to explain the lattice parameter and density increases at high As pressure. These considerations imply that the antistructure disorder should be considered in the future point defect models. Second, the conflict regarding the nature of the prismatic dislocation loop observed by transmission electron microscope in annealed GaAs doped with donor impurities remains. Is the prismatic loop interstitial like or vacancy like? If both are possible under what conditions the formation of one type is favoured over the other?

The nature and origin of point defects involved in the climb of dark-line defects during degradation of $\mathrm{GaAs}$ lasers are still unknown. The climb process in GaAs requires point defect in equal supersaturation in both sublattice. Since this is rather unlikely, Petroff and Kimerling (1976) proposed a new model in which supersaturation of only one point defect is needed for the dislocation climb. While they proposed climb by the absorption of interstitials O'Hara et al (1977) and Hutchinson et al (1978) suggested that climb occurs by the emission of vacancies. Further, the role of anti-site defects, dissociations of dislocations and the different types of dislocations are factors still not considered in the climb process. 
There is at present no satisfactory way of distinguishing between the shuffe set and the glide set dislocations in a particular experiment. This introduces a difficulty in deducing the electrical nature of the dislocations. Further since the core structure of the $\alpha$ and $\beta$ dislocations are different and that the $\alpha$ dislocation in the shuffle set corresponds to the $\beta$ dislocation in the glide set, point defectdislocation interaction effects would be different for the two sets. Recent high resolution electron microscopy experiments by Olsen and Spence (1981) to distinguish the glide and shuffle sets are encouraging in this respect.

Finally, annealing experiments on high purity GaAs samples as a function of As pressure are needed to find whether donors or acceptors are created at the low As side. Care must be taken to prevent $\mathrm{Cu}$ contamination in these annealing experiments. It is also necessary to combine two or more techniques to analyse the same sample. In this respect, new experimental techniques may have to be employed. The recent success with EPR experiments (Wagner et al 1980) raises the hope for more direct evidence for point defects. To a limited extent positron lifetime measurements have also been used (Gopinathan et al 1977; Cheng et al 1979 ) and they implicate $V_{G a}$ related defects. Positron lifetime together with $2 y$ angular correlation measurements would be helpful. Recently, Mössbauer spectroscopy has also been used to study Sn implanted GaAs (Holm and Weyer 1980) giving evidence for Sn-vacancy complexes. Ballistic phonon attenuation measurements in $\mathrm{Ga}_{1-\mathrm{x}} \mathrm{Al} \times \mathrm{As}$ (Narayanamurti et al 1979) substantiated the impurity - $V_{\mathrm{As}}$ model for the DX centres. The potential of internal friction measurements has not yet been fully utilized in GaAs. Perhaps in the future these measurements would be expected to be employed to understand and control the defect structure of GaAs.

\section{Acknowledgements}

The author gratefully acknowledges many helpful discussions with G A Baraff, S Mahajan, J R Patel, P M Petroff and W R Wagner. He is indebted to B V Dutt, L C Kimerling, S Mahajan and P M Petroff for their helpful comments on the manuscript. The author would also like to thank $N$ E Schumaker for his kind support and encouragement during preparation of this review.

\section{References}

Albers M, Gabriel A and Schroter W 1977 Radiation Effects in Semiconductors. Inst. Phys. Conf. Ser. 31 (London Inst. Phys.) p. 509

Alexander H 1979 J. Phys. Colloq. (France) 40 C-6 p. 1

Alexander H and Haasen P 1968 Solid State Phys. (eds.) F Seitz and D Turnbull (New York: Academic Press) 2227

Anthony P J, Hartman R L, Schumaker N E and Wagner W R 1982 J. Appl. Phys. 53756

Arnold G W 1969 Phys. Rev. 183777

Ashen D J, Dean P J, Hurle D T J, Mullen J B and White A M 1975 J. Phys. Chem. Solids 361041 
Astakhov V M, Vasileva L F, Sidorov Yu G and Stenin S I 1980 Sov. Phys. Solid State 22279

Bachelet G B, Baraff GA, and Schluter M 1981 Phys. Rev. B24 915

Baitinger U, Arndt J and Schnepf D 1969 J. Mater. Sci. 4396

Ball R K and Hurchinson P W $1980 \mathrm{~J}$. Mater. Sci. 152376

Baraff G A, Kane E O and Schluter M 1980 Phys. Rev. B21 5662

Barrera J 1975 Proc. 5th Biennial Cornell Electrical Eng. Conf. (New York : Cornell University Press) p. 135

Batavin V V and Popova G V 1974 Sov. Phys. Semicond. 71194

Bell R L, Latkowski R and Willoughby A F W 1966 J. Mater. Sci. 166

Belyatskaya N S, Grishina S P, Lopatin E V, Milvidskii M G, Osvenskii V B and Formin V G 1972. Sov. Phys. Crystallogr. 17126

Berholc J and Pantelides S T 1978. Phys. Rev. B18 1780

Bhattacharya P K, Ku J W, Owen S J T, Aebiv T, Cooper III C B and Moon R L 1980 Appl. Phys. Lett. 36304

Birey H and Sites J 1980 J. Appl. Phys. 51619

Blanc J 1975 Philos. Mag. 321023

Bohm K and Fischer B 1979 J. Appl. Phys. 505453

Bohm K and Gwinner D 1978 Appl. Phys. 17155

Brailovskii E. Groza A and Broudnyi V N 1973 Inst. Phys. Conf. Series 16121

Brice J C 1970 J. Cryst. Growth 79

Brice J C and King G D 1966 Nature (London') 2091346

Bublık V T, Karataev, Kulagin R S, Milvidskii M G, Osvenskii V B, Stolyarov O G and Kholodnyi L P 1973 Sov. Phys. Crystollogr. 18218

Bois and Pinard 1974 Phys. Rev. B9 4171

Casey Jr. H C and Panish M B 1978 Heterostructure lasers Part B (New York Academy : New York)

Chakraverty B K and Dreyfus R W 1966 J. Appl. Phys. 37631

Chang L L, Esaki L and Tsu R 1971 Appl. Phys. Lett. 19143

Cheng L J, Karins J P, Corbett J W and Kimerling L C 1979 J. Appl. Phys. 502962

Chiang S Y and Pearson G L 1975a J. Appl. Phys. 462986

Chiang S Y and Pearson G L 1975b J. Lumines 10313

Choi S K, Mihara M and Ninomiya T 1977 Jpn. J. Appl. Phys. 16737

Cockayne D J H, Ray I L F and Whelan M J 1969 Philos. Mag. 201265

Corbett J W and Bourgoin J C 1975 Point defects in solids (eds) Jr. J H Crawford and L M Slifkin (New York: Plenum) Vol. 2 p. 1

Driscoll C M H, Willoughby A F W and Bellamy B A J 1971 J. Mater. Sci. 61389

Driscoll C M H and Willoughby A F W 1972 Inst. Phys. Conf. Ser. 16 (London Inst. of Phys.) p. 377

Esquivel A L, Sen S and Lin W N 1976 J. Appl. Phys. 472588

Ettenberg M 1974 J. Appl. Phys. 45901

Ettenterg M, Olsen G H and Nuesse C J 1976 Appl. Phys. Lett. 29141

Far vacque J L and Ferre D 1980 Rev. Phys. Appl. 1533

Fazzio A, Leite J R and DeSiqueira M L 1979 J. Phys. C. Solid State 123469

Figielski T 1980 Czech. J. Phys. B30 318

Frank W and Gosele U 1980 Appl. Phys. 23303

Gatos H C, Finn M C and Lavine M C 1961 J. Appl. Phys. 321174

Glimchuck K D and Prokhorovich A V 1975 Phys. Status Solidi A29 339

Goldstein B 1961 Phys. Rev. 1211305

Gomez A and Hirsch P B 1978 Philos. Mag. A38 733

Gopinathan K P, Sundar C S, Swaminathan V and Viswanathan B 1977 Nucl. Solid State Phys. (India). C 20232

Gottschalk H, Patzer G and Alexander H 1978 Phys. Stat. Solidi. 45207

Gwinner D and Labusch R 1979 J. Phys. Colloq. (France) 40 C-6 75

Haasen P 1957 Acta Metall. 5598

Haasen P 1975 Phys. Stat. Solidi A28 145

Hasegawa F and Majerfield A 1976 Electron Lett. 1252 
Heinke W and Queisser H J 1974 Phys. Rev. Lett. 331082

Hirsch P B 1979a J. Phys. Collog. (France) 40 C-6 27

Hirsch P B 1979b J. Phys. Colloq. (France) p. 117

Hirsch P B 1981 Defects in semiconductors (eds) J Narayan and T Y Tan (North Holland Pub. Co.: New York) p. 257

Hirsch P B, Howie A, Nicholson R B, Pashley D W and Whelan M J 1965 Electron microscopy of thin crystals (London; Butterworths)

Hirth J P and Lothe J 1968 Theory of dislocation (New York : McGraw Hill)

Holm N E and Weyer G 1980 J. Phys. C. Solid State 131109

Holt D B 1962 J. Phys. Chem. Solids 231353

Hornstra J 1958 J. Phys. Chem. Solids 5129

Hughes B and Narayanan G H 1978 Phys. Stat. Solidi. A46 627

Hurle D T J 1979 J. Phys. Chem. Solids 40613627639647

Hutchinson P W and Dobson P S 1974 Philos. Mag. 3065

Hutchinson P W and Dobson P S 1975a J. Mater. Sci. 101636

Hutchinson P W and Dobson P S 1975b Philos. Mag. 32745

Hutchinson P W, Dobson P S, Wakefield B and O'Hara S 1978 Solid State Elect. 211413

Hutchinson P W and Dobson P S 1980 Philos. Mag. A41 601

Hwang C J 1968 J. Appl. Phys. 394307

Hwang C J 1969 Phys. Rev. 180827

Ikoma H 1970 Jpn. J. Phys. Soc. 281474

lkoma $H$ and Nakagawa M 1972 Jpn. J. Appl. Phys. 11338

lkoma H and Wang S S 1969 Jpn. J. Phys. Soc. 27512

Jl'in N P and Masterov 1976 Sov. Phys. Semicond. 10496

Imai H, Fugiwara T, Segi K, Takusagawa $M$ and Takanashi H 1979 Jpn. J. Appl. Phys. 18589

Ishida T, Maeda K and Takeuchi S 1980 Appl. Phys. 21257

Itoh T and Takeuchi M 1977 Jpn. J. Appl. Phys. 16227

Jaros M and Brand S 1976 Phys. Rev. B1 44494

Jeong M U, Shirafuji J and Inuishi Y 1973 Jpn. J. Appl. Phys. 12109

Jones R 1979 J. Phys. Colloq. (France) 40 C-6 33

Jordan A S, Caruso R and VonNeida A R 1980 Bell. Syst. Tech. J. 59593

Kamejima T, Ishida K and Matsui J 1977 Jpn. J. Appl. Phys. 16233

Kamejima T, Matsui J, Seki Y and Watanabe H 1979 J. Appl. Phys. 503312

Kasano H 1978a J. Appl. Phys. 494021

Kasano H 1978b J. Appl. Phys. 494746

Kimerling L C and Lang D V 1975 Inst. of Phys. Conf. Series 23 (London Inst. of Phys.) p. 589

Kimerling L C and Patel J R 1979 Appl. Phys. Lett. 3473

Kirkby P A 1975 IEEE J. Quant. Elect. 11562

Klein P B, Nordquist P E R and Srebenmann P G 1980 J. Appl. Phys. 514861

Kroger F A 1974 The chemistry of imperfect crystals (North Holland Pub. Co. : Amsterdam) Vol. 2

Kroger F A 1977 Ann. Rev. Mater Sci. 7449

Kroger F A and Vink H J 1956 Solid State Phys. (eds.) F Seitz and D Turnbull (New York Academy) Vol. 3307

Kryukova I V, Malysheva O V, Petrushenko Yu V, Smirnov V A, Stolyarov O G, Tityunik L Nand Shniger V E 1974 Sov. J. Quant. Electro. 4378

Laister D and Jenkins G M 1971 Philos. Mag. 231077

Laister D and Jenkins G M 1973 J. Mater. Sci. 81218

Labusch R and Schroter W 1980 in Dislocations in solids (ed.) F R N Nabarro (Amsterdam : North Holland Pub. Ca.) Vol. 5 p. 127

Lang D V 1976 in Radiation effects in semiconductors (eds) N B Urli and J W Corbett Inst. Phys. Conf. Ser. 31 (London Inst. of Phys.) p. 70

Lang D V, Logan R A and Kimerling L C 1977 Phys. Rev. B15 4874

Lang D V, Logan R A and Jaros M 1979a Phy. Rev. B19 1015 
Lang D V and Logan R A 1979 Proc. 14th Intl. Conf. Phys. Semicond. Inst. of Phys. Conf. Series 43433

Lang D V, Petroff P M, Logan R A and Johnston Jr W D 1979b Phys. Rev. Lett. 421353

Lin A L and Bube R H 1975 J. Appl. Phys. 465302

Lin M S, Gamo K, Masuda K and Namba S 1975 Jpn. J. Appl. Phys. 141839

Logan R A and Hurle D T J 1971 J. Phys. Chem. Solids. 321739

Lum W Y, Wieder H H, Koschel WH, Bishop S G and McCombe B D 1977 Appl. Phys. Lett. 301

Mader S and Blakeslee A E 1974 Appl. Phys. Lett. 25365

Marklund S 1979 Phys. Stat. Solidi B92 83

Matsui J, Ishida K and Nannichi Y 1975 Jpn. J. Appl. Phys. 141555

Miller M D. Olsen G H and Ettenberg M 1977 Appl. Phys. Lett. 31538

Monemar B and Woolhouse G R 1976 Appl. Phys. Lett. 29605

Morgulis L M, Milvidskii M G and Osvenskii V B 1974 Sov. Phys. Solid State 16784

Munoz E, Snyder W L and Moll J L 1970 Appl. Phys. Lett. 16262

Nakata $H$ and Ninomiya T 1977 Jpn. J. Phys. Soc. 42552

Nakata H and Ninomiya T 1979 Jpn. J. Phys. Soc. 471912

Narayanamurti V, Logan R A and Chin M A 1979 Phys. Rev. Lett. 431536

Narayanan G H and Kachare A H 1975 Phys. Status Solidi A26 657

Nishizawa J, Otsuka H, Yamakoshi S and Ishido K 1974 Jpn. J. Appl. Phys. 1346

Nishizawa S, Sinozaki and Ishida K $1973 \mathrm{~J}$. Appl. Phys. 441638

Norris B and Narayanan G H 1977 J. Appl. Phys. 482784

Oberg S 1978 Phys. Status Solidi B89 K127

O'Hara S, Hutchinson P W and Dobson P S 1977 Appl. Phys. Lett. 30368

Olsen A and Spence J C H 1981 Philos. Mag. A43 945

Osvenskii V B and Eremeev V V 1971 Sov. Phys. Crystollogr. 15906

Osvenskii V B. Kholodnyi L P and Milvidskii M G 1972 Sov. Phys. Solid State 131790

Osvenskii V B, Proshko G P and Grekova S N 1971 Sov. Phys. Solid. State 13. 1409

Otsubo M, Miki H and Mitsui S 1977 Jpn. J. Appl. Phys. 161957

Palfrey H D and Willoughby A F W 1980 Electrochem. Soc Meeting, St. Louis

Patel J R and Testardi L R. 1977 Appl. Phys. Lett, 303

Patel J R, Testardi L R and Freeland P E 1976 Phys. Rev B13 3548

Petroff P M 1975 in Lattice defects in semiconductors, Inst. of Phys. Conf. Ser. (London : Inst of Phys.) Vol. 23, p. 73

Petroff P M 1981 in Defects in semiconductors (eds) J Narayan and T Y Tan (Amsterdam: North Holland) p. 457

Petroff P M and Hartman R L 1974 J. Appl. Phys. 453899

Petroff P M and Kimerling L C 1976 Appl. Phys. Lett. 29461

Petroff. P M, Logan R A and Savage A 1980 Phys 4 Rev. Lett. 44287

Pois and Pinard 1974 Phys. Rev. 134171

Pons D, Mircea A and Bourgoin J $1980 \mathrm{~J}$. Appl. Phys 514150

Potts H R and Pearson G L 1966 J Appl. Phys 372098

Rao E V K and Duhamel N 1978 J. Appl. Phys. 493457

Read W T 1954 Philos. Mag. 45775

Roberts F E 1965 Phys. Lett. 1721

Roedel R J, von Neida A R, Caruso R and Dawson L R 1979 J. Electrochem. Soc. 126637

Romano-Moran R and Ashley K L 1973 J. Phys. Chem. Sol. 34427

Rytova N S and Fistul V I 1970 Sov. Phys. Semicond. 4939

Sazbin N P, Milvidskii M G, Osvenskii V B and Stolyarov O G 1966 Sov. Phys. Sol. State 81223

Sch röter W, Labusch R and Haasen P 1977 Phys. Rev. B15 4121

Shockley W 1953 Phys. Rev. 91228

Spitzer W G and Allred 1968 Appl. Phys. Lett. 125

Stacy W T and DeKock A J R 1978 J. Elect. Mater. 7708

Swaminathan V 1975 Mechanical behaviour of GaAs Single crystals. Ph.D Thesis, University of Southern Califcrnia (Unpublished) 
Swaminathan V and Copley S M 1975a J. Am. Ceram. Soc. 58482

Swaminathan V and Copley S M 1975b J. Appl. Phys. 474405

Swaminathan V, Kimerling L C and Wagner W R 1981 a Appl. Phys. Lett. 38881

Swaminathan V, Schumaker N E, Zilko J L, Wagner W R and Parsons C A 1981 b J. Appl. Phys. 52412

Swaminathan V and Tsang W T 1981c Appl. Phys. Lett. 38347

Swaminathan V, Schumaker $N$ E and Zilko $J$ L 1981d J. Lumines. 22153

Umeno M, Kawabe H and Doi K 1979 Philos. Mag. 39183

Van Vechten J A 1975 J. Electrochem. Soc. 122423

Vorobkalo F M, Glinchuk K D, Prokorovich AV and John G 1973 Phys. Status Solidi A15 287

Vovnenko V I, Glinchuk K D and Prokorovich A V 1979 Sov. Phys. Semicond. 131062

Wagner W R 1977 GaAs and related compounds. Inst. of Phys. Conf. Ser. (London : Inst. of Phys.) B33 p. 65

Wagner W R 1978 J. Appl. Phys. 49173

Wagner R J, Krebs J J, Stauss G H and White A M 1980 Solid State Commun. 3615

Wakefield B 1979 J. Appl. Phys. 597914

Wakins G D 1975 Lattice defects in semiconductors, Inst. of Phys. Conf. Ser. (London : Inst. of Phys.) Vol. 23, p. 1

Watkins G D and Troxell J R 1980 Phys. Rev. Lett. 44593

Weber E and Alexander $\mathbf{H} 1977$ in Radiation effects in semiconductors, Inst. of Phys. Conf. Ser. (London : Inst. of Phys.) Vol. 31, p. 266

Williams E W and Bebb H B 1972 Semiconductors and semimetals (eds) R K Willardson and A C Beer (New York : Academic Press) Vol. 8, p. 321. 